\title{
Second-order charge currents and stress tensor in a chiral system
}

\author{
Shi-Zheng Yang $\odot,{ }^{1, *}$ Jian-Hua Gao, ${ }^{2, \dagger}$ Zuo-Tang Liang, ${ }^{1, *}$ and Qun Wang ${ }^{3,4,8}$ \\ ${ }^{1}$ Key Laboratory of Particle Physics and Particle Irradiation (MOE), Institute of Frontier and \\ Interdisciplinary Science, Shandong University, Qingdao, Shandong 266237, China \\ ${ }^{2}$ Shandong Provincial Key Laboratory of Optical Astronomy and Solar-Terrestrial Environment, Institute \\ of Space Sciences, Shandong University, Weihai, Shandong 264209, China \\ ${ }^{3}$ Interdisciplinary Center for Theoretical Study and Department of Modern Physics, University of Science \\ and Technology of China, Hefei, Anhui 230026, China \\ ${ }^{4}$ Peng Huanwu Center for Fundamental Theory, Hefei, Anhui 230026, China
}

(Received 4 May 2020; revised 11 December 2020; accepted 14 December 2020; published 31 December 2020)

\begin{abstract}
We study Wigner equations for massless spin-1/2 charged fermions at global equilibrium in static and uniform vorticity and electromagnetic fields. The Wigner functions can be solved order by order from Wigner equations through the power expansion of the vorticity and electromagnetic fields. The nondissipative charge currents and the stress tensor up to the second order can be obtained from Wigner functions. The charge and energy densities and the pressure have contributions from vorticity and electromagnetic fields at the second order. The vector and axial Hall currents can be induced along the direction orthogonal to vorticity and electromagnetic fields at the second order. We also find that the trace anomaly emerges naturally in renormalization of the stress tensor by including quantum corrections from electromagnetic fields.
\end{abstract}

DOI: 10.1103/PhysRevD.102.116024

\section{INTRODUCTION}

It is well known in classical electrodynamics that the electromagnetic field can generate electric currents, such as Olm's current from electric fields or Hall's current from magnetic fields. There are also currents from quantum effects that attract broad interest in high energy nuclear physics and condensed matter physics. One example is chiral anomaly, a pure quantum effect, in which currents along the external magnetic field can be induced; it is called the chiral magnetic effect (CME) [1-3]. The vorticity in an ideal fluid behaves like a magnetic field. Similar to CME, the vorticity can induce the electric current in a charged fluid of massless fermions, which is called the chiral vortical effect (CVE) [4-7]. In addition to CME and CVE, chiral currents can also be generated by vorticity and magnetic fields, these are called the chiral separate effect (CSE) [8,9] or the local polarization effect (LPE) [10]. Theoretical studies of these effects have been carried

\footnotetext{
yangshizheng@mail.sdu.edu.cn

gaojh@sdu.edu.cn

*liang@sdu.edu.cn

§qunwang@ustc.edu.cn
}

Published by the American Physical Society under the terms of the Creative Commons Attribution 4.0 International license. Further distribution of this work must maintain attribution to the author(s) and the published article's title, journal citation, and DOI. Funded by SCOAP ${ }^{3}$. out within a variety of approaches, such as AdS/CFT duality [11-20], relativistic hydrodynamics [21-24], quantum field theory [2,3,25-35], and chiral kinetic theories [10,36-49].

From the point of view of hydrodynamics, these anomalous currents are nondissipative without entropy production and they all appear at the first order in space-time derivatives. It has been shown [50-55] that the relativistic hydrodynamical equations with only first order term are acausal and unstable. This issue can be repaired by including second order terms. We also need to include higher order contributions when vorticity or electromagnetic fields are strong enough. This is the case in high energy heavy ion collisions, in which both strong magnetic fields [56-58] and vorticity fields [59-65] are generated in noncentral collisions. There have been already some earlier attempts to study transport phenomena at the second order in chiral systems including second order hydrodynamics with reversal invariance [24], Kubo formula or diagrammatic methods from the quantum field theory [66-69], chiral kinetic theories [70-73], and equilibrium partition functions or AdS/CFT dualities [74-77].

The Lorentz covariant and gauge invariant quantum transport theories [78-81] based on Wigner functions can be derived from quantum field theory and are expected to include all quantum corrections. In previous works $[10,82]$ by some of us, a power expansion in space-time derivatives and weak fields for Wigner functions of chiral 
fermions was proposed near equilibrium. It turns out that the Wigner function formalism is successful to reproduce first order currents in CME, CVE, CSE, and LPE. In this paper, we will use the power expansion method to derive second order nondissipative charge currents and energymomentum tensor in a noninteracting fluid. The novelty of nondissipative currents is that they are present in global equilibrium. This provides a unique shortcut to investigate these effects because the calculation will be greatly simplified in global equilibrium. However in order to arrive at nontrivial global equilibrium, we must choose static and uniform vorticity and electromagnetic fields. Besides, we will neglect the fermion mass (chiral limit) and make chiral limit, which is valid when the temperature is much greater than the particle's static mass.

In Sec. II, we give a brief overview of the Wigner function formalism for a chiral fermion system. In Sec. III, we solve the equations for the covariant Wigner function at global equilibrium with static and uniform vorticity and electromagnetic fields by using the method of Refs. $[10,48,82,83]$. We give the simplest solution to the Wigner function up to the second order of the vorticity and electromagnetic field. In Secs. IV and $\mathrm{V}$, we present the induced vector and axial currents and energy-momentum tensor up to the second order. It can be verified that the charge (vector current) conservation and the anomalous conservation of axial current hold automatically. There is no infrared and ultraviolet divergence for the vector and axial charges. For the energy-momentum tensor at the second order, the contribution from the vorticity only and that from the vorticity and electromagnetic field are both finite, while the contribution from the electromagnetic field has logarithmic ultraviolet divergence when the Dirac sea or vacuum contribution is included. With a proper dimension regularization, we obtain the results that satisfy the energy-momentum conservation. Especially, after we renormalize the stress tensor by including the quantum correction from the electromagnetic field, the trace anomaly emerges naturally. In Sec. VI, we verify the conservation of the electric charge and the energy momentum as well as the anomalous conservation of the axial charge. In Sec. VI, we extend the special solution to general ones and compare with previous results obtained in Ref [68,69]. A summary of our results is made in Sec. VIII.

We use the convention for the metric tensor $g_{\mu \nu}=$ $\operatorname{diag}(1,-1,-1,-1)$ and the Levi-Civita tensor $\epsilon^{0123}=1$. For notational simplicity, the electric charge of the fermion is absorbed into the vector potential $A^{\mu}$.

\section{WIGNER FUNCTION FORMALISM}

The Wigner function $W(x, p)$ for Dirac fermions is a $4 \times 4$ matrix and is defined as the ensemble average of the Wigner operator [78-80],

$$
\begin{aligned}
W_{\alpha \beta}(x, p)= & \int \frac{d^{4} y}{(2 \pi)^{4}} e^{-i p \cdot y} \\
& \times\left\langle\bar{\psi}_{\beta}\left(x+\frac{y}{2}\right) U\left(x+\frac{y}{2}, x-\frac{y}{2}\right) \psi_{\alpha}\left(x-\frac{y}{2}\right)\right\rangle,
\end{aligned}
$$

where $U$ denotes the gauge link along the straight line between $x-y / 2$ and $x+y / 2$,

$U\left(x+\frac{y}{2}, x-\frac{y}{2}\right) \equiv \operatorname{Exp}\left(-i \int_{x-y / 2}^{x+y / 2} d z^{\mu} A_{\mu}(z)\right)$.

We will restrict ourselves to a system of chiral fermions without collisions in a constant external electromagnetic field $F^{\mu \nu}$ in space and time, i.e., $\partial^{\lambda} F^{\mu \nu}=0$, hence we have removed the path ordering of the gauge link. The Wigner equation for chiral fermions in a constant electromagnetic field is given by [80]

$$
\gamma_{\mu}\left(p^{\mu}+\frac{i}{2} \nabla^{\mu}\right) W(x, p)=0,
$$

where $\gamma^{\mu}$ are Dirac matrices, and $\nabla^{\mu} \equiv \partial_{x}^{\mu}-F^{\mu \nu} \partial_{\nu}^{p}$ with $\partial_{x}\left(\partial^{p}\right)$ is the derivative with respect to $x(p)$. Since the Wigner equation is derived from the Dirac equation, the bilinear operator in the Wigner function should not be normal ordered. It has been demonstrated in Ref. [84] that this feature plays a central role to give rise to the chiral anomaly in quantum kinetic theory. We can decompose the Wigner function in terms of 16 independent generators of the Clifford algebra,

$W=\frac{1}{4}\left[\mathcal{F}+i \gamma^{5} \mathcal{P}+\gamma^{\mu} \mathcal{V}_{\mu}+\gamma^{5} \gamma^{\mu} \mathcal{A}_{\mu}+\frac{1}{2} \sigma^{\mu \nu} \mathcal{S}_{\mu \nu}\right]$,

where we have suppressed arguments of the Wigner function for notational simplicity.

For chiral fermions, it is more convenient to define the chiral component

$$
\mathcal{J}_{s}^{\mu} \equiv \frac{1}{2}\left(\mathcal{V}^{\mu}+s \mathcal{A}^{\mu}\right)
$$

with $s=+1$ and -1 corresponding to the right-hand and left-hand component, respectively. Substituting Esq. (2.4) and (2.5) into Eq. (2.3), we find that the right-hand or lefthand component are decoupled from other components and satisfy

$$
\begin{gathered}
\nabla_{\mu} \mathcal{J}_{s}^{\mu}=0 \\
p_{\mu} \mathcal{J}_{s}^{\mu}=0 \\
p_{\mu} \mathcal{J}_{s \nu}-p_{\nu} \mathcal{J}_{s \mu}=-\frac{s}{2} \epsilon_{\mu \nu \rho \sigma} \nabla^{\rho} \mathcal{J}_{s}^{\sigma} .
\end{gathered}
$$


We will suppress the subscript $s$ in Sec. III for notational simplicity and recover it in Sec. IV.

\section{WIGNER FUNCTION NEAR EQUILIBRIUM}

We assume that both the space-time derivative $\partial_{x}$ and the field strength $F^{\mu \nu}$ in the operator $\nabla_{\mu}$ are small variables of the same order and play the role of expansion parameters. We solve the Wigner equation by the covariant perturbation method developed in Refs. [10,48,83] and present the solution near equilibrium up to the second order in $\partial_{x}$ and $F^{\mu \nu}$. In fact, this expansion is equivalent to an expansion in the Planck constant $\hbar$ (or the semiclassical expansion) because $\hbar$ always comes with $\nabla_{\mu}$. According to the perturbation method, the Wigner function can be obtained order by order,

$$
\mathcal{J}_{\mu}=\mathcal{J}_{\mu}^{(0)}+\mathcal{J}_{\mu}^{(1)}+\mathcal{J}_{\mu}^{(2)}+\cdots,
$$

where the superscripts $(0),(1), \ldots$ denote the orders of the power in the expansion. Substituting this expansion into Wigner equations from (2.6) to (2.8) and requiring that the equations hold order by order. The equations for $\mathcal{J}_{\mu}^{(n)}$ with $n>0$ read

$$
\begin{gathered}
\nabla_{\mu} \mathcal{J}^{(n) \mu}=0 \\
p_{\mu} \mathcal{J}^{(n) \mu}=0 \\
p_{\mu} \mathcal{J}_{\nu}^{(n)}-p_{\nu} \mathcal{J}_{\mu}^{(n)}=-\frac{s}{2} \epsilon_{\mu \nu \rho \sigma} \nabla^{\rho} \mathcal{J}^{(n-1) \sigma} .
\end{gathered}
$$

If we define $\mathcal{J}^{(-1) \sigma}=0$, Eq. (3.4) also works for $n=0$. When we contract both sides of Eq. (3.4) with $p^{\nu}$, we have

$$
p^{2} \mathcal{J}_{\mu}^{(n)}=\frac{s}{2} \epsilon_{\mu \nu \rho \sigma} p^{\nu} \nabla^{\rho} \mathcal{J}^{(n-1) \sigma},
$$

where we have used Eq. (3.3). Hence the general form of $\mathcal{J}_{\mu}^{(n)}$ is

$$
\mathcal{J}_{\mu}^{(n)}=J_{\mu}^{(n)} \delta\left(p^{2}\right)+\frac{s}{2 p^{2}} \epsilon_{\mu \nu \rho \sigma} p^{\nu} \nabla^{\rho} \mathcal{J}^{(n-1) \sigma},
$$

where $J_{\mu}^{(n)}$ is nonsingular at $p^{2}=0$. This expression is an iterative equation connecting the $n$th order solution with the $(n-1)$ th order one. The constraint condition (3.3) gives

$$
p^{\mu} J_{\mu}^{(n)} \delta\left(p^{2}\right)=0
$$

In general, we can decompose $J_{\mu}^{(n)}$ into two parts

$$
J_{\mu}^{(n)}(x, p)=p_{\mu} f^{(n)}(x, p)+X_{\mu}^{(n)}(x, p),
$$

where the first term satisfies Eq. (3.7) automatically due to $p^{2} \delta\left(p^{2}\right)=0$ and the second term is assumed to satisfy $p^{\mu} X_{\mu}^{(n)}=0$ when there is no mass-shell constraint.

It is straightforward to write down the zeroth order solution,

$$
\mathcal{J}_{\mu}^{(0)}(x, p)=p_{\mu} f(x, p) \delta\left(p^{2}\right),
$$

without $X_{\mu}^{(0)}$ component. We note that in the above expression we have suppressed the superscript (0) in $f$ because we will set all higher order contributions $f^{(n)}$ for $n \geq 1$ vanish before Sec. VII in which all possible solutions for $f^{(1)}$ and $f^{(2)}$ will be discussed. Substituting the expression (3.9) into Eq. (3.2) with $n=0$ gives the kinetic equation at the zeroth order

$$
\delta\left(p^{2}\right) p^{\mu} \nabla_{\mu} f(x, p)=0 .
$$

Since we try to obtain the solution near equilibrium, at the zeroth order we can choose $f$ as the Fermi-Dirac distribution function,

$$
\begin{array}{r}
f=\frac{1}{4 \pi^{3}} \frac{1}{e^{\beta \cdot p-\bar{\mu}_{s}}+1}, \quad\left(p_{0}>0\right), \\
f=\frac{1}{4 \pi^{3}}\left(\frac{1}{e^{-\beta \cdot p+\bar{\mu}_{s}}+1}-1\right), \quad\left(p_{0}<0\right),
\end{array}
$$

where

$\beta^{\mu} \equiv \beta u^{\mu}=\frac{u^{\mu}}{T}, \quad \bar{\mu}_{s} \equiv \frac{\mu_{s}}{T}=\bar{\mu}+s \bar{\mu}_{5}, \quad \bar{\mu}=\frac{\mu}{T}, \quad \bar{\mu}_{5}=\frac{\mu_{5}}{T}$,

with $u$ being the fluid four-velocity, $T$ the temperature, $\mu_{s}$ the right-hand/left-hand chemical potential, $\mu$ the vector chemical potential, and $\mu_{5}$ the axial chemical potential. We can always introduce the axial chemical potential in the zeroth order solution for chiral fermions because the axial current is always conserved when there is no electromagnetic field at the zeroth order. Actually we can even introduce the axial chemical potential when the electromagnetic field is present because we can redefine the conserving axial current by absorbing the Chern-Simons current. The total current is conserved and we can introduce the chemical potential corresponding to this conserved charge. In the solution given in Eqs. (3.11) and (3.12), we see that $\mathcal{J}_{\mu}^{(0)}(x, p)$ or $f(x, p)$ depends on $x$ only through $u(x), T(x), \mu(x)$ and $\mu_{5}(x)$. The Dirac sea (or vacuum) contribution -1 in the antiparticle distribution $[85,86]$ is indispensable because there is no normal ordering in the definition of the Wigner function (2.1). With the distribution (3.11) and (3.12), the Wigner function $\mathcal{J}_{\mu}^{(0)}$ is in the form 


$$
\mathcal{J}_{\mu}^{(0)}=\frac{p_{\mu}}{4 \pi^{3}}\left[\frac{1}{e^{\beta \cdot p-\bar{\mu}_{s}}+1} \frac{\delta\left(p_{0}-|\mathbf{p}|\right)}{2|\mathbf{p}|}+\left(\frac{1}{e^{-\beta \cdot p+\bar{\mu}_{s}}+1}-1\right) \frac{\delta\left(p_{0}+|\mathbf{p}|\right)}{2|\mathbf{p}|}\right]
$$

Inserting Eqs. (3.11) or (3.12) into the kinetic equation (3.10) we obtain

$$
\delta\left(p^{2}\right) p^{\mu} \nabla_{\mu} f=\delta\left(p^{2}\right) f^{\prime}\left[\frac{1}{2} p^{\mu} p^{\nu}\left(\partial_{\mu} \beta_{\nu}+\partial_{\nu} \beta_{\mu}\right)-p^{\mu} \partial_{\mu} \bar{\mu}-p^{\mu} F_{\mu \nu} \beta^{\nu}-s p^{\mu} \partial_{\mu} \bar{\mu}_{5}\right]=0,
$$

where we have used the shorthand notation $f^{\prime} \equiv \partial f$ / $\partial(\beta \cdot p)$. It is obvious that when the constraint conditions

$$
\begin{gathered}
\partial_{\mu} \beta_{\nu}+\partial_{\nu} \beta_{\mu}=0, \\
\partial_{\mu} \bar{\mu}+F_{\mu \nu} \beta^{\nu}=0, \\
\partial_{\mu} \bar{\mu}_{5}=0,
\end{gathered}
$$

are all satisfied, the Wigner function (3.9) with (3.11) and (3.12) are indeed the solution to Eq. (3.10). These conditions are actually global equilibrium conditions for the system under static and uniform vorticity and electromagnetic fields. General solutions to these constraint conditions are

$$
\begin{gathered}
\beta_{\mu}=-\Omega_{\mu \nu} x^{\nu}, \\
\bar{\mu}=-\frac{1}{2} F^{\mu \lambda} x_{\lambda} \Omega_{\mu \nu} x^{\nu}+c, \\
\bar{\mu}_{5}=c_{5},
\end{gathered}
$$

together with the integrability condition

$$
F_{\lambda}{ }^{\mu} \Omega^{\nu \lambda}-F_{\lambda}{ }^{\nu} \Omega^{\mu \lambda}=0
$$

where $\Omega^{\mu \nu}$ and $c_{5} / c$ are constant antisymmetric tensor and constants, respectively. The integrability condition is obtained by differentiating both sides of Eq. (3.17) with $\partial_{\nu}$ and applying the commutativity of partial derivatives

$$
\partial_{\nu} \partial_{\mu} \bar{\mu}=\partial_{\mu} \partial_{\nu} \bar{\mu} \Rightarrow F_{\mu \lambda} \partial_{\nu} \beta^{\lambda}=F_{\nu \lambda} \partial_{\mu} \beta^{\lambda},
$$

which leads to the condition (3.22) directly with Eq. (3.19). It should be noted that $\Omega_{\mu \nu}$ is nothing but the thermal vorticity tensor of the fluid

$$
\Omega_{\mu \nu}=\frac{1}{2}\left(\partial_{\mu} \beta_{\nu}-\partial_{\nu} \beta_{\mu}\right) .
$$

Substituting the zeroth order solution (3.9) into Eq. (3.6) with $n=1$ gives rise to the first order solution

$$
\begin{aligned}
\mathcal{J}_{\mu}^{(1)} & =J_{\mu}^{(1)} \delta\left(p^{2}\right)+\frac{s}{2 p^{2}} \epsilon_{\mu \nu \rho \sigma} p^{\nu} \nabla^{\rho} \mathcal{J}^{(0) \sigma}, \\
& =X_{\mu}^{(1)} \delta\left(p^{2}\right)+s \tilde{F}_{\mu \nu} p^{\nu} f \delta^{\prime}\left(p^{2}\right)
\end{aligned}
$$

where we have dropped the term proportional to $p_{\mu} \delta\left(p^{2}\right)$ and used $\tilde{F}^{\mu \nu}=(1 / 2) \epsilon^{\mu \nu \rho \sigma} F_{\rho \sigma}$ and $\delta^{\prime}(x)=-(1 / x) \delta(x)$. The unknown $X_{\mu}^{(1)}$ can be further constrained by inserting Eq. (3.25) into Eq. (3.4)

$$
\begin{array}{r}
\left(p_{\mu} X_{\nu}^{(1)}-p_{\nu} X_{\mu}^{(1)}\right) \delta\left(p^{2}\right)=\frac{s}{2} \epsilon_{\mu \nu \lambda \rho} p^{\lambda} \nabla^{\rho} f \delta\left(p^{2}\right), \\
=-\frac{s}{2}\left(p_{\mu} \tilde{\Omega}_{\nu \lambda} p^{\lambda}-p_{\nu} \tilde{\Omega}_{\mu \lambda} p^{\lambda}\right) f^{\prime} \delta\left(p^{2}\right),
\end{array}
$$

where $\tilde{\Omega}_{\mu \nu}=(1 / 2) \epsilon_{\mu \nu \rho \sigma} \Omega^{\rho \sigma}$. In order to arrive at the last equation, we have used the specific distribution (3.11)(3.12) and conditions (3.16)-(3.18). Obviously, from the equation above, we can set

$$
X_{\mu}^{(1)}=-\frac{s}{2} \tilde{\Omega}_{\mu \lambda} p^{\lambda} f^{\prime},
$$

which results in

$\mathcal{J}_{\mu}^{(1)}=-\frac{s}{2} \tilde{\Omega}_{\mu \lambda} p^{\lambda} f^{\prime} \delta\left(p^{2}\right)+\frac{s}{2} \epsilon_{\mu \nu \rho \sigma} p^{\nu} F^{\rho \sigma} f \delta^{\prime}\left(p^{2}\right)$.

Under global equilibrium conditions (3.16)-(3.18), it is straightforward to verify that above $\mathcal{J}_{\mu}^{(1)}$ given above automatically satisfies Eq. (3.2) with $n=1$. This means that Eq. (3.28) is indeed the solution of the first order under global equilibrium conditions.

Now let us turn to the second order solution that has not been considered before. Similar to the way how we obtain the first order solution from the zeroth order, the second order solution can be given by the iterative equation (3.6) with the first order solution (3.28),

$$
\begin{aligned}
\mathcal{J}_{\mu}^{(2)}= & J_{\mu}^{(2)} \delta\left(p^{2}\right)+\frac{s}{2 p^{2}} \epsilon_{\mu \nu \rho \sigma} p^{\nu} \nabla^{\rho} \mathcal{J}^{(1) \sigma}, \\
= & X_{\mu}^{(2)} \delta\left(p^{2}\right)+\frac{1}{4 p^{2}}\left(p_{\mu} \Omega_{\gamma \beta} p^{\beta}-p^{2} \Omega_{\gamma \mu}\right) \Omega^{\gamma \lambda} p_{\lambda} f^{\prime \prime} \delta\left(p^{2}\right) \\
& +\frac{2}{p^{6}}\left(p_{\mu} F_{\gamma \beta} p^{\beta}-p^{2} F_{\gamma \mu}\right) F^{\gamma \lambda} p_{\lambda} f \delta\left(p^{2}\right) \\
& +\frac{1}{p^{4}}\left(p_{\mu} F_{\gamma \beta} p^{\beta}-p^{2} F_{\gamma \mu}\right) \Omega^{\gamma \lambda} p_{\lambda} f^{\prime} \delta\left(p^{2}\right)
\end{aligned}
$$

Here $X_{\mu}^{(2)}$ can be constrained by inserting Eq. (3.29) into Eq. (3.4) with $n=2$. It turns out that 


$$
\left(p_{\mu} X_{\nu}^{(2)}-p_{\nu} X_{\mu}^{(2)}\right) \delta\left(p^{2}\right)=0
$$

which leads to $X_{\nu}^{(2)}=0$, where we have used conditions (3.16)-(3.18) once again to arrive at the final result. Now we finally obtain the second order solution

$$
\begin{aligned}
\mathcal{J}_{\mu}^{(2)}= & -\frac{1}{4} \Omega_{\gamma \mu} \Omega^{\gamma \lambda} p_{\lambda} f^{\prime \prime} \delta\left(p^{2}\right)-\frac{1}{4} p_{\mu} \Omega_{\gamma \beta} p^{\beta} \Omega^{\gamma \lambda} p_{\lambda} f^{\prime \prime} \delta^{\prime}\left(p^{2}\right) \\
& +F_{\gamma \mu} \Omega^{\gamma \lambda} p_{\lambda} f^{\prime} \delta^{\prime}\left(p^{2}\right)+\frac{1}{2} p_{\mu} F_{\gamma \beta} p^{\beta} \Omega^{\gamma \lambda} p_{\lambda} f^{\prime} \delta^{\prime \prime}\left(p^{2}\right) \\
& -F_{\gamma \mu} F^{\gamma \lambda} p_{\lambda} f \delta^{\prime \prime}\left(p^{2}\right)-\frac{1}{3} p_{\mu} F_{\gamma \beta} p^{\beta} F^{\gamma \lambda} p_{\lambda} f \delta^{\prime \prime \prime}\left(p^{2}\right),
\end{aligned}
$$

where we have used the identity

$$
p^{6} \delta^{\prime \prime \prime}\left(p^{2}\right)=-3 p^{4} \delta^{\prime \prime}\left(p^{2}\right)=6 p^{2} \delta^{\prime}\left(p^{2}\right)=-6 \delta\left(p^{2}\right) .
$$

\section{VECTOR AND AXIAL CURRENTS}

Once we have the Wigner function in phase space, the right-handed or left-handed current can be obtained directly by integrating corresponding components of the Wigner function over the four momentum

$$
j_{s}^{\mu}=\int d^{4} p \mathcal{J}_{s}^{\mu}
$$

where we have recovered the chirality index $s$. The vector and axial currents are given by

$$
j^{\mu}=j_{+1}^{\mu}+j_{-1}^{\mu}, \quad j_{5}^{\mu}=j_{+1}^{\mu}-j_{-1}^{\mu} .
$$

Note that the vector current can also be called the fermion number or charge current, while the axial current can also be called the chiral charge or chiral current. The results for the zeroth and first order current are well known

$$
\begin{gathered}
j_{s}^{(0) \mu}=n_{s} u^{\mu}, \\
j_{s}^{(1) \mu}=\xi_{s} \omega^{\mu}+\xi_{B s} B^{\mu},
\end{gathered}
$$

where $n_{s}$ is the fermion number density, and $\xi_{s}$ and $\xi_{B s}$ are transport coefficients associated with $\mathrm{CVE}$ and $\mathrm{CME}$, respectively, in the right-handed and left-handed current $j_{s}^{\mu}$. They are given by

$$
\begin{gathered}
n_{s}=\frac{\mu_{s}}{6 \pi^{2}}\left(\pi^{2} T^{2}+\mu_{s}^{2}\right), \\
\xi_{s}=\frac{s}{12 \pi^{2}}\left(\pi^{2} T^{2}+3 \mu_{s}^{2}\right), \\
\xi_{B s}=\frac{s}{4 \pi^{2}} \mu_{s} .
\end{gathered}
$$

In the zeroth order result $n_{s}$, we have dropped the infinite vacuum contribution. In Eq. (4.3) the vorticity vector $\omega^{\mu}$ and the magnetic field vector $B^{\mu}$ are defined from the decomposition

$$
\begin{gathered}
F_{\mu \nu}=E_{\mu} u_{\nu}-E_{\nu} u_{\mu}+\epsilon_{\mu \nu \rho \sigma} u^{\rho} B^{\sigma}, \\
T \Omega_{\mu \nu}=\varepsilon_{\mu} u_{\nu}-\varepsilon_{\nu} u_{\mu}+\epsilon_{\mu \nu \rho \sigma} u^{\rho} \omega^{\sigma},
\end{gathered}
$$

with

$$
E^{\mu}=F^{\mu \nu} u_{\nu}, \quad B^{\mu}=\tilde{F}^{\mu \nu} u_{\nu}=\frac{1}{2} \epsilon^{\mu \nu \alpha \beta} u_{\nu} F_{\alpha \beta},
$$

$\varepsilon^{\mu}=T \Omega^{\mu \nu} u_{\nu}, \quad \omega^{\mu}=T \tilde{\Omega}^{\mu \nu} u_{\nu}=\frac{1}{2} \epsilon^{\mu \nu \alpha \beta} u_{\nu} \partial_{\alpha}^{x} u_{\beta}$.

Similar to the electric or magnetic component of $F_{\mu \nu}$, it is convenient to name $\varepsilon_{\mu}$ and $\omega_{\mu}$ as the electric vorticity and the magnetic vorticity, respectively. It follows that the vector and axial current are given by

$$
\begin{gathered}
j^{(0) \mu}=n u^{\mu}, \\
j^{(1) \mu}=\xi \omega^{\mu}+\xi_{B} B^{\mu}, \\
j_{5}^{(0) \mu}=n_{5} u^{\mu}, \\
j_{5}^{(1) \mu}=\xi_{5} \omega^{\mu}+\xi_{B 5} B^{\mu},
\end{gathered}
$$

with

$$
\begin{aligned}
n & =\frac{\mu}{3 \pi^{2}}\left(\pi^{2} T^{2}+\mu^{2}+3 \mu_{5}^{2}\right), \quad n_{5}=\frac{\mu_{5}}{3 \pi^{2}}\left(\pi^{2} T^{2}+3 \mu^{2}+\mu_{5}^{2}\right), \\
\xi & =\frac{\mu \mu_{5}}{\pi^{2}}, \quad \xi_{B}=\frac{\mu_{5}}{2 \pi^{2}}, \quad \xi_{5}=\frac{1}{6 \pi^{2}}\left[\pi^{2} T^{2}+3\left(\mu^{2}+\mu_{5}^{2}\right)\right], \\
\xi_{B 5} & =\frac{\mu}{2 \pi^{2}} .
\end{aligned}
$$

where $n$ and $n_{5}$ are the fermion number (charge) and chiral charge density, respectively, and $\xi, \xi_{B}, \xi_{5}$, and $\xi_{B 5}$ are wellknown anomalous transport coefficients associated with CVE, CME, LPE, and CSE, respectively.

The second order current can be obtained by integrating Eq. (3.31) over the four momentum, 


$$
\begin{aligned}
j_{s}^{(2) \mu}= & -\frac{1}{4} \Omega^{\gamma \mu} \Omega_{\gamma \lambda} u^{\lambda} \int d^{4} p(u \cdot p) f_{s}^{\prime \prime} \delta\left(p^{2}\right)-\frac{1}{4} u^{\mu} u^{\beta} u_{\lambda} \Omega_{\gamma \beta} \Omega^{\gamma \lambda} \int d^{4} p(u \cdot p)^{3} f_{s}^{\prime \prime} \delta^{\prime}\left(p^{2}\right) \\
& -\frac{1}{12}\left(\Delta^{\mu \beta} u_{\lambda}+\Delta^{\mu}{ }_{\lambda} u^{\beta}+\Delta_{\lambda}{ }^{\beta} u^{\mu}\right) \Omega_{\gamma \beta} \Omega^{\gamma \lambda} \int d^{4} p(u \cdot p) \bar{p}^{2} f_{s}^{\prime \prime} \delta^{\prime}\left(p^{2}\right) \\
& +F^{\gamma \mu} \Omega_{\gamma \lambda} u^{\lambda} \int d^{4} p(u \cdot p) f_{s}^{\prime} \delta^{\prime}\left(p^{2}\right)+\frac{1}{2} u^{\mu} u^{\beta} u_{\lambda} F_{\gamma \beta} \Omega^{\gamma \lambda} \int d^{4} p(u \cdot p)^{3} f_{s}^{\prime} \delta^{\prime \prime}\left(p^{2}\right) \\
& +\frac{1}{6}\left(\Delta^{\mu \beta} u_{\lambda}+\Delta^{\mu}{ }_{\lambda} u^{\beta}+\Delta_{\lambda}{ }^{\beta} u^{\mu}\right) F_{\gamma \beta} \Omega^{\gamma \lambda} \int d^{4} p(u \cdot p) \bar{p}^{2} f_{s}^{\prime} \delta^{\prime \prime}\left(p^{2}\right) \\
& -F^{\gamma \mu} F_{\gamma \lambda} u^{\lambda} \int d^{4} p(u \cdot p) f_{s} \delta^{\prime \prime}\left(p^{2}\right)-\frac{1}{3} u^{\mu} u^{\beta} u_{\lambda} F_{\gamma \beta} F^{\gamma \lambda} \int d^{4} p(u \cdot p)^{3} f_{s} \delta^{\prime \prime \prime}\left(p^{2}\right) \\
& -\frac{1}{9}\left(\Delta^{\mu \beta} u_{\lambda}+\Delta^{\mu}{ }_{\lambda} u^{\beta}+\Delta_{\lambda}{ }^{\beta} u^{\mu}\right) F_{\gamma \beta} F^{\gamma \lambda} \int d^{4} p(u \cdot p) \bar{p}^{2} f_{s} \delta^{\prime \prime \prime}\left(p^{2}\right) .
\end{aligned}
$$

In the above equation we have used following moment identities

$$
\begin{gathered}
\int d^{4} p p^{\lambda} Y=u^{\lambda} \int d^{4} p(u \cdot p) Y, \\
\int d^{4} p p^{\mu} p^{\beta} p^{\lambda} Y=u^{\mu} u^{\beta} u^{\lambda} \int d^{4} p(u \cdot p)^{3} Y+\frac{1}{3}\left(\Delta^{\mu \beta} u^{\lambda}+\Delta^{\mu \lambda} u^{\beta}+\Delta^{\lambda \beta} u^{\mu}\right) \int d^{4} p(u \cdot p) \bar{p}^{2} Y,
\end{gathered}
$$

where $Y$ can be any scalar functions of $u \cdot p$ and $p^{2}, \Delta^{\mu \nu}=g^{\mu \nu}-u^{\mu} u^{\nu}$, and $\bar{p}^{\mu}=\Delta^{\mu \nu} p_{\nu}$. Using the decomposition (4.8) and (4.9), $j_{s, \mu}^{(2)}$ can be put into the form

$$
\begin{aligned}
j_{s}^{(2) \mu}= & u^{\mu}\left(\varepsilon^{2}+\omega^{2}\right) \frac{1}{6} \int d^{4} p(u \cdot p) \bar{p}^{2} f_{s}^{\prime \prime} \delta^{\prime}\left(p^{2}\right) \\
& -\epsilon^{\gamma \mu \rho \sigma} \varepsilon_{\rho} u_{\sigma} \omega_{\gamma} \int d^{4} p(u \cdot p) f_{s}^{\prime \prime}\left[\frac{1}{6} \bar{p}^{2} \delta^{\prime}\left(p^{2}\right)+\frac{1}{4} \delta\left(p^{2}\right)\right] \\
& -u^{\mu}(\varepsilon \cdot E+\omega \cdot B) \frac{1}{3} \int d^{4} p(u \cdot p) \bar{p}^{2} f_{s}^{\prime} \delta^{\prime \prime}\left(p^{2}\right) \\
& +\epsilon^{\gamma \mu \rho \sigma} E_{\rho} u_{\sigma} \omega_{\gamma} \int d^{4} p(u \cdot p) f_{s}^{\prime}\left[\frac{1}{3} \bar{p}^{2} \delta^{\prime \prime}\left(p^{2}\right)+\delta^{\prime}\left(p^{2}\right)\right] \\
& +u^{\mu}\left(E^{2}+B^{2}\right) \frac{2}{9} \int d^{4} p(u \cdot p) \bar{p}^{2} f_{s} \delta^{\prime \prime \prime}\left(p^{2}\right) \\
& -\epsilon^{\gamma \mu \rho \sigma} u_{\rho} B_{\sigma} E_{\gamma} \int d^{4} p(u \cdot p) f_{s}\left[\frac{2}{9} \bar{p}^{2} \delta^{\prime \prime \prime}\left(p^{2}\right)+\delta^{\prime \prime}\left(p^{2}\right)\right] .
\end{aligned}
$$

After completing integrals in Eq. (4.20), we obtain second order currents

$$
\begin{aligned}
j_{s}^{(2) \mu}= & -\frac{\mu_{s}}{4 \pi^{2}}\left(\varepsilon^{2}+\omega^{2}\right) u^{\mu}-\frac{1}{8 \pi^{2}}(\varepsilon \cdot E+\omega \cdot B) u^{\mu}-\frac{C_{s}}{24 \pi^{2}}\left(E^{2}+B^{2}\right) u^{\mu} \\
& -\frac{1}{8 \pi^{2}} \epsilon^{\mu \nu \rho \sigma} u_{\nu} E_{\rho} \omega_{\sigma}-\frac{C_{s}}{12 \pi^{2}} \epsilon^{\mu \nu \rho \sigma} u_{\nu} E_{\rho} B_{\sigma},
\end{aligned}
$$

where

$$
C_{s}=\frac{1}{T} \int_{0}^{\infty} \frac{d p_{0}}{p_{0}}\left[\frac{e^{p_{0} / T-\bar{\mu}_{s}}}{\left(e^{p_{0} / T-\bar{\mu}_{s}}+1\right)^{2}}-\frac{e^{p_{0} / T+\bar{\mu}_{s}}}{\left(e^{p_{0} / T+\bar{\mu}_{s}}+1\right)^{2}}\right] .
$$

It is obvious that $C_{s}$ is an odd function of $\bar{\mu}_{s}$. When $\left|\bar{\mu}_{s}\right| \ll 1$ or at high temperature limit, we can expand the integrand in Eq. (4.22) in serials and work out the integral analytically 


$$
C_{s}=-\frac{14 \zeta^{\prime}(-2) \mu_{s}}{T^{2}} \approx \frac{0.4263 \mu_{s}}{T^{2}}
$$

When $\left|\bar{\mu}_{s}\right| \gg 1$ or at low temperature limit, we can approximate the Fermi-Dirac distribution function by a step function $\Theta\left( \pm \mu_{s}-p_{0}\right)$ and obtain the analytic result

$$
C_{s}=\frac{1}{\mu_{s}} .
$$

From Eq. (4.21) the vector and axial current are given by

$$
\begin{aligned}
j^{(2) \mu}= & -\frac{\mu}{2 \pi^{2}}\left(\varepsilon^{2}+\omega^{2}\right) u^{\mu}-\frac{1}{4 \pi^{2}}(\varepsilon \cdot E+\omega \cdot B) u^{\mu}-\frac{C}{12 \pi^{2}}\left(E^{2}+B^{2}\right) u^{\mu} \\
& -\frac{1}{4 \pi^{2}} \epsilon^{\mu \nu \rho \sigma} u_{\nu} E_{\rho} \omega_{\sigma}-\frac{C}{6 \pi^{2}} \epsilon^{\mu \nu \rho \sigma} u_{\nu} E_{\rho} B_{\sigma}, \\
j_{5}^{(2) \mu}= & -\frac{\mu_{5}}{2 \pi^{2}}\left(\varepsilon^{2}+\omega^{2}\right) u^{\mu}-\frac{C_{5}}{12 \pi^{2}}\left(E^{2}+B^{2}\right) u^{\mu}-\frac{C_{5}}{6 \pi^{2}} \epsilon^{\mu \nu \rho \sigma} u_{\nu} E_{\rho} B_{\sigma},
\end{aligned}
$$

with

$$
C=\frac{1}{2}\left(C_{+1}+C_{-1}\right), \quad C_{5}=\frac{1}{2}\left(C_{+1}-C_{-1}\right) .
$$

When $\left|\mu_{s}\right| \ll T$, we have

$$
C=-\frac{14 \zeta^{\prime}(-2) \mu}{T^{2}} \approx \frac{0.4263 \mu}{T^{2}}, \quad C_{5}=-\frac{14 \zeta^{\prime}(-2) \mu_{5}}{T^{2}} \approx \frac{0.4263 \mu_{5}}{T^{2}} .
$$

When $\left|\mu_{s}\right| \gg T$, we have

$$
C=\frac{\mu}{\left(\mu^{2}-\mu_{5}^{2}\right)}, \quad C_{5}=-\frac{\mu_{5}}{\left(\mu^{2}-\mu_{5}^{2}\right)} .
$$

Now we look closely at the vector current (4.25). The first line of (4.25) indicates that the charge density is modified by quadratic terms $\varepsilon^{2}, \omega^{2}, E^{2}, B^{2}, \varepsilon \cdot E$, and $\omega \cdot B$. The second line of (4.25) are the Hall currents induced along the direction orthogonal to both $E^{\mu}$ and $\omega^{\nu}$ or that orthogonal to both $E^{\mu}$ and $B^{\nu}$ in the comoving frame of the fluid cell. It is interesting to observe that there is no Hall current induced by $\varepsilon^{\mu}$ and $\omega^{\nu}$. It should be clarified here that the mixed Hall current $\epsilon^{\mu \nu \rho \sigma} u_{\nu} E_{\rho} \omega_{\sigma}$ is actually identical to $\epsilon^{\mu \nu \rho \sigma} u_{\nu} \varepsilon_{\rho} B_{\sigma}$ in this paper due to the integrability condition (3.22), which is equivalent to

$\epsilon_{\mu \nu \rho \sigma}\left(E^{\rho} \omega^{\sigma}-\varepsilon^{\rho} B^{\sigma}\right)=0 \quad$ and $\quad \epsilon_{\mu \nu \rho \sigma}\left(E^{\rho} \varepsilon^{\sigma}+\omega^{\rho} B^{\sigma}\right)=0$.

For the axial current, the first and second terms in (4.26) indicate that the axial charge density gets modified by quadratic terms $\varepsilon^{2}, \omega^{2}, E^{2}$, and $B^{2}$ but not from mixed terms $\varepsilon \cdot E$ and $\omega \cdot B$ due to the symmetry which is different from the charge density. The last term in (4.26) is the axial Hall current generated by $E^{\mu}$ and $B^{\nu}$ only, but not by $E^{\mu}$ and $\omega^{\nu}$ or $\varepsilon^{\mu}$ and $B^{\nu}$, which is also different from the vector current. Like the charge current, there is no axial Hall current from $\varepsilon^{\mu}$ and $\omega^{\nu}$.

\section{ENERGY-MOMENTUM TENSOR}

In the Wigner function formalism, the stress or energymomentum tensor can be from the vector component as

$$
T^{\mu \nu}=\int d^{4} p \mathcal{V}^{\mu} p^{\nu}=\int d^{4} p\left(\mathcal{J}_{+1}^{\mu}+\mathcal{J}_{-1}^{\mu}\right) p^{\nu} .
$$

Note that this is the canonical definition and is not necessarily symmetric. The results for the stress tensor of the right-handed or left-handed part at the zeroth and first order are

$$
\begin{aligned}
T_{s}^{(0) \mu \nu}= & \int d^{4} p \mathcal{J}_{s}^{(0) \mu} p^{\nu}=u^{\mu} u^{\nu} \rho_{s}-\frac{1}{3} \Delta^{\mu \nu} \rho_{s}, \\
T_{s}^{(1) \mu \nu}= & \int d^{4} p \mathcal{J}_{s}^{(1) \mu} p^{\nu}, \\
= & s n_{s}\left(u^{\mu} \omega^{\nu}+u^{\nu} \omega^{\mu}\right) \\
& +\frac{\xi_{s}}{2}\left(u^{\mu} B^{\nu}+u^{\nu} B^{\mu}-\epsilon^{\mu \nu \alpha \beta} u_{\alpha} E_{\beta}\right) \\
& -\frac{s n_{s}}{2}\left(u^{\mu} \omega^{\nu}-u^{\nu} \omega^{\mu}+\epsilon^{\mu \nu \alpha \beta} u_{\alpha} \varepsilon_{\beta}\right),
\end{aligned}
$$


where $n_{s}$ and $\xi_{s}$ in $T_{s}^{(1) \mu \nu}$ are given by Eqs. (4.5), (4.6), and the energy density $\rho_{s}$ in $T_{s}^{(0) \mu \nu}$ is

$$
\rho_{s}=\frac{T^{4}}{2 \pi^{2}}\left(\frac{7}{60} \pi^{4}+\frac{1}{2} \pi^{2} \bar{\mu}_{s}^{2}+\frac{1}{4} \bar{\mu}_{s}^{4}\right),
$$

where we have dropped the infinite vacuum energy density. After taking a sum of the right-handed and left-handed contributions, we obtain the total energy-momentum tensor

$$
\begin{aligned}
& T^{(0) \mu \nu}=T_{+1}^{(0) \mu \nu}+T_{-1}^{(0) \mu \nu}=\rho u^{\mu} u^{\nu}-\frac{1}{3} \rho \Delta^{\mu \nu} \\
T^{(1) \mu \nu}= & T_{+1}^{(0) \mu \nu}+T_{-1}^{(0) \mu \nu} \\
= & n_{5}\left(u^{\mu} \omega^{\nu}+u^{\nu} \omega^{\mu}\right)+\frac{\xi}{2}\left(u^{\mu} B^{\nu}+u^{\nu} B^{\mu}-\epsilon^{\mu \nu \alpha \beta} u_{\alpha} E_{\beta}\right) \\
& -\frac{n_{5}}{2}\left(u^{\mu} \omega^{\nu}-u^{\nu} \omega^{\mu}+\epsilon^{\mu \nu \alpha \beta} u_{\alpha} \varepsilon_{\beta}\right)
\end{aligned}
$$

where $n$ and $\xi$ in $T^{(1) \mu \nu}$ are given by Eq. (4.16), and the energy density in $T^{(0) \mu \nu}$ is

$\rho=\frac{T^{4}}{4 \pi^{2}}\left[\frac{7}{15} \pi^{4}+2 \pi^{2}\left(\bar{\mu}^{2}+\bar{\mu}_{5}^{2}\right)+\bar{\mu}^{4}+6 \bar{\mu}^{2} \bar{\mu}_{5}^{2}+\bar{\mu}_{5}^{4}\right]$.

Now let us compute the stress tensor at the second order. We decompose the stress tensor into three parts,

$$
T_{s}^{(2) \mu \nu}=T_{s, \mathrm{vv}}^{(2) \mu \nu}+T_{s, \mathrm{ve}}^{(2) \mu \nu}+T_{s, \mathrm{ee}}^{(2) \mu \nu},
$$

which "v" means the vorticity and "e" means the electromagnetic field, so these three terms are coupling terms of the vorticity-vorticity, vorticity-electromagnetic-field, and electromagnetic-field-electromagnetic-field, respectively. These terms are given by

$$
\begin{aligned}
T_{s, \mathrm{VV}}^{(2) \mu \nu} & =-\frac{1}{4} \Omega_{\beta}^{\gamma} \Omega_{\gamma \lambda} \int d^{4} p p^{\mu} p^{\nu} p^{\beta} p^{\lambda} f^{\prime \prime} \delta^{\prime}\left(p^{2}\right)-\frac{1}{4} \Omega^{\gamma \mu} \Omega_{\gamma \lambda} \int d^{4} p p^{\nu} p^{\lambda} f^{\prime \prime} \delta\left(p^{2}\right), \\
T_{s, \mathrm{ve}}^{(2) \mu \nu} & =\frac{1}{2} F_{\beta}^{\gamma} \Omega_{\gamma \lambda} \int d^{4} p p^{\mu} p^{\nu} p^{\beta} p^{\lambda} f^{\prime} \delta^{\prime \prime}\left(p^{2}\right)+F^{\gamma \mu} \Omega_{\gamma \lambda} \int d^{4} p p^{\nu} p^{\lambda} f^{\prime} \delta^{\prime}\left(p^{2}\right), \\
T_{s, \mathrm{ee}}^{(2) \mu \nu} & =-\frac{1}{3} F_{\beta}^{\gamma} F_{\gamma \lambda} \int d^{4} p p^{\mu} p^{\nu} p^{\beta} p^{\lambda} f \delta^{\prime \prime \prime}\left(p^{2}\right)-F^{\gamma \mu} F_{\gamma \lambda} \int d^{4} p p^{\nu} p^{\lambda} f \delta^{\prime \prime}\left(p^{2}\right) .
\end{aligned}
$$

Using moment identities

$$
\begin{gathered}
\int d^{4} p p^{\nu} p^{\lambda} Y=u^{\nu} u^{\lambda} \int d^{4} p(u \cdot p)^{2} Y+\frac{1}{3} \Delta^{\mu \nu} \int d^{4} p \bar{p}^{2} Y, \\
\int d^{4} p p^{\mu} p^{\nu} p^{\beta} p^{\lambda} Y= \\
u^{\mu} u^{\nu} u^{\beta} u^{\lambda} \int d^{4} p(u \cdot p)^{4} Y+\frac{1}{15}\left(\Delta^{\mu \nu} \Delta^{\beta \lambda}+\Delta^{\mu \beta} \Delta^{\nu \lambda}+\Delta^{\mu \lambda} \Delta^{\beta \nu}\right) \int d^{4} p \bar{p}^{4} Y \\
+\frac{1}{3}\left(u^{\mu} u^{\nu} \Delta^{\beta \lambda}+u^{\beta} u^{\lambda} \Delta^{\mu \nu}+u^{\mu} u^{\beta} \Delta^{\nu \lambda}+u^{\mu} u^{\lambda} \Delta^{\beta \nu}\right. \\
\left.+u^{\nu} u^{\lambda} \Delta^{\beta \mu}+u^{\nu} u^{\beta} \Delta^{\mu \lambda}\right) \int d^{4} p(u \cdot p)^{2} \bar{p}^{2} Y,
\end{gathered}
$$

and the decomposition (4.8) and (4.9), we can write the first two equations as

$$
\begin{aligned}
T_{s, \mathrm{VV}}^{(2) \mu \nu}= & -\frac{\beta^{2}}{4} u^{\mu} u^{\nu} \varepsilon^{2} \int d^{4} p(u \cdot p)^{4} f^{\prime \prime} \delta^{\prime}\left(p^{2}\right)-\frac{\beta^{2}}{60}\left[\Delta^{\mu \nu}\left(\varepsilon^{2}-4 \omega^{2}\right)+2 \varepsilon^{\mu} \varepsilon^{\nu}+2 \omega^{\mu} \omega^{\nu}\right] \int d^{4} p \bar{p}^{4} f^{\prime \prime} \delta^{\prime}\left(p^{2}\right) \\
& -\frac{\beta^{2}}{12}\left[u^{\mu} u^{\nu}\left(\varepsilon^{2}-2 \omega^{2}\right)+\Delta^{\mu \nu} \varepsilon^{2}+2\left(u^{\mu} \epsilon^{\nu \alpha \beta \gamma}+u^{\nu} \epsilon^{\mu \alpha \beta \gamma}\right) u_{\alpha} \varepsilon_{\beta} \omega_{\gamma}\right] \int d^{4} p(u \cdot p)^{2} \bar{p}^{2} f^{\prime \prime} \delta^{\prime}\left(p^{2}\right) \\
& -\frac{\beta^{2}}{4}\left(u^{\mu} u^{\nu} \varepsilon^{2}+u^{\nu} \epsilon^{\mu \alpha \beta \gamma} u_{\alpha} \varepsilon_{\beta} \omega_{\gamma}\right) \int d^{4} p(u \cdot p)^{2} f^{\prime \prime} \delta\left(p^{2}\right) \\
& -\frac{\beta^{2}}{12}\left(\varepsilon^{\mu} \varepsilon^{\nu}+\omega^{\mu} \omega^{\nu}-\Delta^{\mu \nu} \omega^{2}+u^{\mu} \epsilon^{\nu \alpha \beta \gamma} u_{\alpha} \varepsilon_{\beta} \omega_{\gamma}\right) \int d^{4} p \bar{p}^{2} f^{\prime \prime} \delta\left(p^{2}\right),
\end{aligned}
$$




$$
\begin{aligned}
T_{s, \mathrm{ve}}^{(2) \mu \nu}= & \beta u^{\mu} u^{\nu} \varepsilon \cdot E \int d^{4} p(u \cdot p)^{4} f^{\prime} \delta^{\prime \prime}\left(p^{2}\right) \\
& +\frac{\beta}{30}\left[\Delta^{\mu \nu}(\varepsilon \cdot E-4 \omega \cdot B)+2 E^{\mu} \varepsilon^{\nu}+2 \omega^{\mu} B^{\nu}\right] \int d^{4} p \bar{p}^{4} f^{\prime} \delta^{\prime \prime}\left(p^{2}\right) \\
& +\frac{\beta}{3}\left[u^{\mu} u^{\nu}(\varepsilon \cdot E-2 \omega \cdot B)+\Delta^{\mu \nu} \varepsilon \cdot E+2\left(u^{\mu} \epsilon^{\nu \alpha \beta \gamma}+u^{\nu} \epsilon^{\mu \alpha \beta \gamma}\right) u_{\alpha} E_{\beta} \omega_{\gamma}\right] \int d^{4} p(u \cdot p)^{2} \bar{p}^{2} f^{\prime} \delta^{\prime \prime}\left(p^{2}\right) \\
& +\beta\left(u^{\mu} u^{\nu} \varepsilon \cdot E+u^{\nu} \epsilon^{\mu \alpha \beta \gamma} u_{\alpha} E_{\beta} \omega_{\gamma}\right) \int d^{4} p(u \cdot p)^{2} f^{\prime} \delta^{\prime}\left(p^{2}\right) \\
& +\frac{\beta}{3}\left(E^{\mu} \varepsilon^{\nu}+\omega^{\mu} B^{\nu}-\Delta^{\mu \nu} \omega \cdot B+u^{\mu} \epsilon^{\nu \alpha \beta \gamma} u_{\alpha} E_{\beta} \omega_{\gamma}\right) \int d^{4} p \bar{p}^{2} f^{\prime} \delta^{\prime}\left(p^{2}\right)
\end{aligned}
$$

Completing integration and collecting similar terms, we obtain

$$
\begin{aligned}
T_{s, \mathrm{vV}}^{(2) \mu \nu}= & -\frac{s}{2} \xi_{s}\left[3 u^{\mu} u^{\nu}\left(\omega^{2}+\varepsilon^{2}\right)-\Delta^{\mu \nu}\left(\omega^{2}+\varepsilon^{2}\right)-2\left(u^{\mu} \epsilon^{\nu \alpha \beta \gamma}+u^{\nu} \epsilon^{\mu \alpha \beta \gamma}\right) u_{\alpha} \varepsilon_{\beta} \omega_{\gamma}\right. \\
& \left.-2\left(u^{\mu} \epsilon^{\nu \alpha \beta \gamma}-u^{\nu} \epsilon^{\mu \alpha \beta \gamma}\right) u_{\alpha} \varepsilon_{\beta} \omega_{\gamma}\right], \\
T_{s, \mathrm{ve}}^{(2) \mu \nu}= & -\frac{s}{2} \xi_{B s}\left[u^{\mu} u^{\nu}(\omega \cdot B+\varepsilon \cdot E)-\left(\omega^{\mu} B^{\nu}+E^{\mu} \varepsilon^{\nu}\right)\right. \\
& \left.-\left(u^{\mu} \epsilon^{\nu \alpha \beta \gamma}+u^{\nu} \epsilon^{\mu \alpha \beta \gamma}\right) u_{\alpha} E_{\beta} \omega_{\gamma}-2\left(u^{\mu} \epsilon^{\nu \alpha \beta \gamma}-u^{\nu} \epsilon^{\mu \alpha \beta \gamma}\right) u_{\alpha} E_{\beta} \omega_{\gamma}\right],
\end{aligned}
$$

However, when we deal with $T_{s, \text { ee }}^{(2) \mu \nu}$, we find that it has logarithmic ultraviolet divergence which has to be regularized and renormalized. The regularization with a naïve momentum cutoff will break Lorentz invariance and destroy the energy momentum conservation. To avoid such a problem, we apply dimensional regularization. Now let us make a tensor decomposition in $d=4-\epsilon$ dimension with a small positive number $\epsilon$.

$$
\begin{aligned}
\int d^{d} p p_{\nu} p_{\lambda} Y & =u_{\nu} u_{\lambda} \int d^{d} p(u \cdot p)^{2} Y+\frac{1}{d-1} \Delta_{\mu \nu} \int d^{d} p \bar{p}^{2} Y \\
\int d^{d} p p_{\mu} p_{\nu} p_{\beta} p_{\lambda} Y= & u_{\mu} u_{\nu} u_{\beta} u_{\lambda} \int d^{d} p(u \cdot p)^{4} Y \\
& +\frac{1}{d^{2}-1}\left(\Delta_{\mu \nu} \Delta_{\beta \lambda}+\Delta_{\mu \beta} \Delta_{\nu \lambda}+\Delta_{\mu \lambda} \Delta_{\beta \nu}\right) \int d^{d} p \bar{p}^{4} Y \\
& +\frac{1}{d-1}\left(u_{\mu} u_{\nu} \Delta_{\beta \lambda}+u_{\beta} u_{\lambda} \Delta_{\mu \nu}+u_{\mu} u_{\beta} \Delta_{\nu \lambda}+u_{\mu} u_{\lambda} \Delta_{\beta \nu}\right. \\
& \left.+u_{\nu} u_{\lambda} \Delta_{\beta \mu}+u_{\nu} u_{\beta} \Delta_{\mu \lambda}\right) \int d^{d} p(u \cdot p)^{2} \bar{p}^{2} Y
\end{aligned}
$$

With such tensor decomposition in $d=4-\epsilon$ dimension, we can write Eq. (5.11) as

$$
\begin{aligned}
T_{s, \mathrm{ee}}^{(2) \mu \nu}= & -\frac{1}{3} u^{\mu} u^{\nu} E^{2} \int d^{4-\varepsilon} p(u \cdot p)^{4} f \delta^{\prime \prime \prime}\left(p^{2}\right) \\
& -\frac{1}{45-24 \varepsilon}\left(\Delta^{\mu \nu} F_{\gamma}^{\beta} F^{\gamma \lambda} \Delta_{\beta \lambda}+2 \Delta^{\mu \kappa} \Delta^{\nu \lambda} F_{\gamma \kappa} F_{\lambda}^{\gamma}\right) \int d^{4-\varepsilon} p \bar{p}^{4} f \delta^{\prime \prime \prime}\left(p^{2}\right) \\
& -\frac{1}{9-3 \varepsilon}\left(u^{\mu} u^{\nu} F_{\gamma}^{\beta} F^{\gamma \lambda} \Delta_{\beta \lambda}+\Delta^{\mu \nu} E^{2}+2 u^{\mu} \Delta^{\nu \lambda} E^{\gamma} F_{\gamma \lambda}+2 u^{\nu} \Delta^{\mu \lambda} E^{\gamma} F_{\gamma \lambda}\right) \\
& \times \int d^{4-\varepsilon} p(u \cdot p)^{2} \bar{p}^{2} f \delta^{\prime \prime \prime}\left(p^{2}\right) \\
& -u^{\nu} F^{\gamma \mu} E_{\gamma} \int d^{4} p(u \cdot p)^{2} f \delta^{\prime \prime}\left(p^{2}\right)-\frac{1}{3-\varepsilon} \Delta^{\nu \lambda} F^{\gamma \mu} F_{\gamma \lambda} \int d^{4-\varepsilon} p \bar{p}^{2} f \delta^{\prime \prime}\left(p^{2}\right)
\end{aligned}
$$

We give details of the integration in the first term as an example in Appendix A. After integrating over momentum, we obtain the pure electromagnetic part of the energy-momentum tensor 


$$
\begin{aligned}
T_{s, \mathrm{ee}}^{(2) \mu \nu}= & -\frac{1}{12} \kappa_{s}^{\epsilon}\left(\frac{1}{4} g^{\mu \nu} F_{\gamma \beta} F^{\gamma \beta}-F^{\gamma \mu} F_{\gamma}{ }^{\nu}\right)+\frac{1}{48 \pi^{2}} u^{\mu} u^{\nu} E^{2}-\frac{1}{48 \pi^{2}} \Delta^{\mu \nu}\left(E^{2}+2 B^{2}\right) \\
& +\frac{1}{12 \pi^{2}}\left(E^{\mu} E^{\nu}+B^{\mu} B^{\nu}\right)+\frac{1}{16 \pi^{2}}\left(u^{\mu} \epsilon^{\nu \alpha \beta \gamma}+u^{\nu} \epsilon^{\mu \alpha \beta \gamma}\right) u_{\alpha} E_{\beta} B_{\gamma} \\
& +\frac{1}{16 \pi^{2}}\left(u^{\mu} \epsilon^{\nu \alpha \beta \gamma}-u^{\nu} \epsilon^{\mu \alpha \beta \gamma}\right) u_{\alpha} E_{\beta} B_{\gamma},
\end{aligned}
$$

where $\kappa_{s}^{\epsilon}$ is given by

$$
\kappa_{s}^{\epsilon}=\frac{4 \pi^{(3-\epsilon) / 2} T^{-\epsilon}}{\Gamma((3-\epsilon) / 2)(2 \pi)^{3-\epsilon}} \int_{0}^{\infty} \frac{d y}{y^{1+\epsilon}}\left[\frac{1}{e^{\left(y-\bar{\mu}_{s}\right)}+1}+\frac{1}{e^{\left(y+\bar{\mu}_{s}\right)}+1}-1\right] .
$$

We can expand $\kappa_{s}^{\epsilon}$ around $\epsilon=0$ as

$$
\kappa_{s}^{\epsilon}=-\frac{1}{\pi^{2}}\left[\frac{1}{\epsilon}+\ln 2+\frac{1}{2} \ln \pi+\frac{1}{2} \psi\left(\frac{3}{2}\right)-\ln T+\hat{\kappa}_{s}\right],
$$

where $\psi(x)$ is the digamma function and $\hat{\kappa}_{s}$ is given by

$$
\hat{\kappa}_{s}=\int_{0}^{\infty} d y \ln y \frac{d}{d y}\left[\frac{1}{e^{\left(y-\bar{\mu}_{s}\right)}+1}+\frac{1}{e^{\left(y+\bar{\mu}_{s}\right)}+1}\right] .
$$

Obviously, we can see in (5.24) that the integral in (5.22) contains logarithmic ultraviolet divergence at the limit $\epsilon \rightarrow 0$. The coefficient $\kappa_{s}^{\epsilon}$ or $\hat{\kappa}_{s}$ is an even function of $\bar{\mu}_{s}$. It is also easy to verify

$$
\left.\epsilon \kappa_{s}^{\epsilon}\right|_{\epsilon \rightarrow 0}=-\frac{1}{\pi^{2}},\left.\quad \frac{d \kappa_{s}^{\epsilon}\left(\bar{\mu}_{s}\right)}{d \beta}\right|_{\epsilon \rightarrow 0}=-\frac{T}{\pi^{2}},\left.\quad \frac{d \kappa_{s}^{\epsilon}\left(\bar{\mu}_{s}\right)}{d \bar{\mu}_{s}}\right|_{\epsilon \rightarrow 0}=T C_{s}\left(\bar{\mu}_{s}\right) .
$$

Taking a sum over contributions from left-handed and right-handed fermions, the total stress tensor is given by

$$
\begin{aligned}
T_{\mathrm{vv}}^{(2) \mu \nu}=- & \frac{1}{2} \xi_{5}\left[3 u^{\mu} u^{\nu}\left(\omega^{2}+\varepsilon^{2}\right)-\Delta^{\mu \nu}\left(\omega^{2}+\varepsilon^{2}\right)-2\left(u^{\mu} \epsilon^{\nu \alpha \beta \gamma}+u^{\nu} \epsilon^{\mu \alpha \beta \gamma}\right) u_{\alpha} \varepsilon_{\beta} \omega_{\gamma}\right. \\
& \left.-2\left(u^{\mu} \epsilon^{\nu \alpha \beta \gamma}-u^{\nu} \epsilon^{\mu \alpha \beta \gamma}\right) u_{\alpha} \varepsilon_{\beta} \omega_{\gamma}\right], \\
T_{\mathrm{ve}}^{(2) \mu \nu}=- & \frac{1}{2} \xi_{B 5}\left[u^{\mu} u^{\nu}(\omega \cdot B+\varepsilon \cdot E)-\left(\omega^{\mu} B^{\nu}+E^{\mu} \varepsilon^{\nu}\right)-\left(u^{\mu} \epsilon^{\nu \alpha \beta \gamma}+u^{\nu} \epsilon^{\mu \alpha \beta \gamma}\right) u_{\alpha} E_{\beta} \omega_{\gamma}\right. \\
- & \left.2\left(u^{\mu} \epsilon^{\nu \alpha \beta \gamma}-u^{\nu} \epsilon^{\mu \alpha \beta \gamma}\right) u_{\alpha} E_{\beta} \omega_{\gamma}\right], \\
T_{\mathrm{ee}}^{(2) \mu \nu}= & -\frac{1}{6} \kappa^{\epsilon}\left(\frac{1}{4} g^{\mu \nu} F_{\gamma \beta} F^{\gamma \beta}-F^{\gamma \mu} F_{\gamma}{ }^{\nu}\right)+\frac{1}{24 \pi^{2}}\left[u^{\mu} u^{\nu} E^{2}-\Delta^{\mu \nu}\left(E^{2}+2 B^{2}\right)\right. \\
& +4\left(E^{\mu} E^{\nu}+B^{\mu} B^{\nu}\right)+3\left(u^{\mu} \epsilon^{\nu \alpha \beta \gamma}+u^{\nu} \epsilon^{\mu \alpha \beta \gamma}\right) u_{\alpha} E_{\beta} B_{\gamma} \\
& \left.+3\left(u^{\mu} \epsilon^{\nu \alpha \beta \gamma}-u^{\nu} \epsilon^{\mu \alpha \beta \gamma}\right) u_{\alpha} E_{\beta} B_{\gamma}\right],
\end{aligned}
$$

where $\kappa^{\epsilon}=\left(\kappa_{+1}^{\epsilon}+\kappa_{-1}^{\epsilon}\right) / 2$.

We see that energy density and pressure are modified in vorticity and electromagnetic fields at the second order, which are not the case at the first order. Similar to the first order result in Eq. (5.6), there are also antisymmetric contributions to the energy-momentum tensor at the second order. It is straightforward to verify with Eqs. (5.5), (5.6), (5.26)-(5.28) that the trace of the total energy-momentum tensor up to the second order vanishes

$$
g_{\mu \nu} T^{\mu \nu}=0,
$$

free of trace anomaly. Note that in taking the trace of $T_{\mathrm{ee}}^{(2) \mu \nu}$ we used $g_{\mu \nu} g^{\mu \nu}=4-\epsilon$. The trace anomaly does not arise here because the electromagnetic field in our work is only a classical background field and keeps scale invariance. We note that the divergent part in $T_{\mathrm{ee}}^{(2) \mu \nu}$ is proportional to the stress tensor of the free electromagnetic field. It is remarkable that this divergent term $\sim 1 / \epsilon$ is exactly the 
contribution from the quantum correction of the electromagnetic field [87] but with a wrong sign. Hence we can add this contribution to our result, cancel the divergent term and arrive at the renormalized finite result

$$
\begin{aligned}
T_{\mathrm{ee}, \operatorname{Re}}^{(2) \mu \nu}= & \frac{1}{6 \pi^{2}}\left(\hat{\kappa}+\ln \frac{\Lambda}{T}\right)\left(\frac{1}{4} g^{\mu \nu} F_{\gamma \beta} F^{\gamma \beta}-F^{\gamma \mu} F_{\gamma}{ }^{\nu}\right) \\
& +\frac{1}{24 \pi^{2}}\left[u^{\mu} u^{\nu} E^{2}-\Delta^{\mu \nu}\left(E^{2}+2 B^{2}\right)\right. \\
& +4\left(E^{\mu} E^{\nu}+B^{\mu} B^{\nu}\right)+3\left(u^{\mu} \epsilon^{\nu \alpha \beta \gamma}+u^{\nu} \epsilon^{\mu \alpha \beta \gamma}\right) u_{\alpha} E_{\beta} B_{\gamma} \\
& \left.+3\left(u^{\mu} \epsilon^{\nu \alpha \beta \gamma}-u^{\nu} \epsilon^{\mu \alpha \beta \gamma}\right) u_{\alpha} E_{\beta} B_{\gamma}\right],
\end{aligned}
$$

where $\hat{\kappa}=\hat{\kappa}_{+1}+\hat{\kappa}_{-1}$ and $\Lambda$ is a renormalization scale in quantum electromagnetic field. Note that we have also absorbed all possible remaining constant terms in Eq. (5.23) into $\Lambda$. After removing the divergence in $T_{\mathrm{ee}}^{(2) \mu \nu}$, we can safely calculate the trace of the energymomentum tensor in four dimensions and obtain the trace anomaly

$$
g_{\mu \nu} T_{\mathrm{ee}, \mathrm{Re}}^{(2)}=\frac{1}{24 \pi^{2}} F_{\mu \nu} F^{\mu \nu}
$$

which originates from the quantum correction of the electromagnetic fields.

In hydrodynamics, we usually express the stress tensor in the Landau frame. In Appendix B, the symmetric part of the stress tensor is written in the Landau frame.

\section{CONSERVATION LAWS}

With $j^{\mu}$ in (4.12), (4.13), and (4.25); $j_{5}^{\mu}$ in (4.14), (4.15), and (4.26); and $T^{\mu \nu}$ in (5.5), (5.6), (5.26), (5.27), and (5.30), we can check conservation laws for these quantities. In doing so, we must restrict ourselves to a specific system in constant and homogeneous electromagnetic fields with conditions (3.16), (3.17), (3.18), and (3.22) or (4.30). Here we will not present a detailed derivation, but give the necessary identities in performing the calculation. These identities hold only under specific conditions that are imposed in this paper:

$$
\begin{gathered}
\partial_{\mu} \frac{u^{\mu}}{T}=0, \quad u \cdot \partial \frac{1}{T}=0, \quad \partial_{\mu} u^{\mu}=0, \quad \partial_{\mu} \frac{1}{T}=-u \cdot \partial \frac{u_{\mu}}{T}=\frac{\varepsilon_{\mu}}{T}, \\
\partial_{\mu} \omega_{\nu}=\varepsilon \cdot \omega g_{\mu \nu}-2 \varepsilon_{\mu} \omega_{\nu}, \\
\partial_{\mu} \varepsilon_{\nu}=\omega_{\mu} \omega_{\nu}-\varepsilon_{\mu} \varepsilon_{\nu}+\varepsilon^{2} u_{\mu} u_{\nu}-\omega^{2} \Delta_{\mu \nu}+\left(u_{\mu} \epsilon_{\nu \lambda \rho \sigma}+u_{\nu} \epsilon_{\mu \lambda \rho \sigma}\right) u^{\lambda} \varepsilon^{\rho} \omega^{\sigma} \\
\partial_{\mu} B_{\nu}=-E_{\mu} \omega_{\nu}+\varepsilon \cdot B u_{\mu} u_{\nu}+\omega \cdot E \Delta_{\mu \nu}-\left(u_{\mu} \epsilon_{\nu \lambda \rho \sigma}+u_{\nu} \epsilon_{\mu \lambda \rho \sigma}\right) u^{\lambda} \varepsilon^{\rho} E^{\sigma}, \\
\partial_{\mu} E_{\nu}=B_{\mu} \omega_{\nu}+\varepsilon \cdot E u_{\mu} u_{\nu}-\omega \cdot B \Delta_{\mu \nu}+\left(u_{\mu} \epsilon_{\nu \lambda \rho \sigma}+u_{\nu} \epsilon_{\mu \lambda \rho \sigma}\right) u^{\lambda} E^{\rho} \omega^{\sigma} .
\end{gathered}
$$

With the help of these identities, we can verify following conservation laws,

$$
\partial^{\mu} j_{\mu}=0, \quad \partial^{\mu} j_{\mu}^{5}=-\frac{1}{2 \pi^{2}} E \cdot B, \quad \partial^{\mu} T_{\mu \nu}=F_{\nu \mu} j^{\mu} .
$$

We note that the second order correction to the axial current does not contribute to the chiral anomaly as it should be. We find that the term proportional to $\ln \Lambda / T$ in Eq. (5.30) is essential to conserve the energy-momentum when the vorticity is present. We can separate the energy-momentum tensor into a symmetric and an antisymmetric part,

$$
T^{\mu \nu}=T_{S}^{\mu \nu}+T_{A}^{\mu \nu}
$$

where the symmetric and antisymmetric part are given by

$$
\begin{aligned}
T_{S}^{\mu \nu}= & \rho u^{\mu} u^{\nu}-\frac{1}{3} \rho \Delta^{\mu \nu}+n_{5}\left(u^{\mu} \omega^{\nu}+u^{\nu} \omega^{\mu}\right)+\frac{\xi}{2}\left(u^{\mu} B^{\nu}+u^{\nu} B^{\mu}\right) \\
& -\frac{1}{2} \xi_{5}\left[3 u^{\mu} u^{\nu}\left(\omega^{2}+\varepsilon^{2}\right)-\Delta^{\mu \nu}\left(\omega^{2}+\varepsilon^{2}\right)-2\left(u^{\mu} \epsilon^{\nu \alpha \beta \gamma}+u^{\nu} \epsilon^{\mu \alpha \beta \gamma}\right) u_{\alpha} \varepsilon_{\beta} \omega_{\gamma}\right] \\
& -\frac{1}{2} \xi_{B 5}\left[u^{\mu} u^{\nu}(\omega \cdot B+\varepsilon \cdot E)-\left(\omega^{\mu} B^{\nu}+E^{\mu} \varepsilon^{\nu}\right)-\left(u^{\mu} \epsilon^{\nu \alpha \beta \gamma}+u^{\nu} \epsilon^{\mu \alpha \beta \gamma}\right) u_{\alpha} E_{\beta} \omega_{\gamma}\right] \\
& +\kappa_{1}^{E} u^{\mu} u^{\nu} E^{2}+\kappa_{1}^{B} u^{\mu} u^{\nu} B^{2}+\kappa_{2}^{E} \Delta^{\mu \nu} E^{2}+\kappa_{2}^{B} \Delta^{\mu \nu} B^{2}+\kappa_{3}\left(E^{\mu} E^{\nu}+B^{\mu} B^{\nu}\right) \\
& +\kappa_{4}\left(u^{\mu} \epsilon^{\nu \alpha \beta \gamma}+u^{\nu} \epsilon^{\mu \alpha \beta \gamma}\right) u_{\alpha} E_{\beta} B_{\gamma},
\end{aligned}
$$




$$
\begin{aligned}
T_{A}^{\mu \nu}= & -\frac{n_{5}}{2}\left(u^{\mu} \omega^{\nu}-u^{\nu} \omega^{\mu}+\epsilon^{\mu \nu \alpha \beta} u_{\alpha} \varepsilon_{\beta}\right)-\frac{\xi}{2} \epsilon^{\mu \nu \alpha \beta} u_{\alpha} E_{\beta}+\xi_{5}\left(u^{\mu} \epsilon^{\nu \alpha \beta \gamma}-u^{\nu} \epsilon^{\mu \alpha \beta \gamma}\right) u_{\alpha} \varepsilon_{\beta} \omega_{\gamma} \\
& +\xi_{B 5}\left(u^{\mu} \epsilon^{\nu \alpha \beta \gamma}-u^{\nu} \epsilon^{\mu \alpha \beta \gamma}\right) u_{\alpha} E_{\beta} \omega_{\gamma}+\frac{1}{8 \pi^{2}}\left(u^{\mu} \epsilon^{\nu \alpha \beta \gamma}-u^{\nu} \epsilon^{\mu \alpha \beta \gamma}\right) u_{\alpha} E_{\beta} B_{\gamma},
\end{aligned}
$$

where we have expressed the energy-momentum tensor in terms of $E_{\mu}$ and $B_{\mu}$ and used the renormalized result in Eq. (5.30). The coefficients are defined as

$$
\begin{aligned}
\kappa_{1}^{E}=-\frac{1}{12 \pi^{2}}\left(\hat{\kappa}+\ln \frac{\Lambda}{T}-\frac{1}{2}\right), & \kappa_{1}^{B}=-\frac{1}{12 \pi^{2}}\left(\hat{\kappa}+\ln \frac{\Lambda}{T}\right), \\
\kappa_{2}^{E}=\frac{1}{12 \pi^{2}}\left(\hat{\kappa}+\ln \frac{\Lambda}{T}-\frac{1}{2}\right), & \kappa_{2}^{B}=\frac{1}{12 \pi^{2}}\left(\hat{\kappa}+\ln \frac{\Lambda}{T}-1\right), \\
\kappa_{3}=-\frac{1}{6 \pi^{2}}\left(\hat{\kappa}+\ln \frac{\Lambda}{T}-1\right), & \kappa_{4}=-\frac{1}{6 \pi^{2}}\left(\hat{\kappa}+\ln \frac{\Lambda}{T}-\frac{3}{4}\right) .
\end{aligned}
$$

We can verify following conservation equations,

$$
\partial_{\mu} T_{S}^{\mu \nu}=F^{\nu \mu} j_{\mu}, \quad \partial_{\mu} T_{A}^{\mu \nu}=0
$$

\section{GENERAL SOLUTION}

We have presented a specific solution in the previous sections by setting $f^{(1)}=f^{(2)}=0$ in Eq. (3.8). The general solution under global equilibrium conditions (3.16)-(3.18) should be a summation of this specific solution and all possible contributions associated with nonvanishing $f^{(1)}$ and $f^{(2)}$. In this section, we determine these contributions. We note that these terms are proportional to $p^{\mu} \delta\left(p^{2}\right)$ and automatically satisfy Eq. (3.3). They can not be further constrained by using Eq. (3.4) because they do not appear on the left-hand side of the equation due to cancellation. Hence the remaining constraint can be only from Eq. (3.2). From Lorentz invariance together with charge and parity invariance, the general $f^{(1)}$ and $f^{(2)}$ must take following forms,

$$
\begin{gathered}
f^{(1)}=(\omega \cdot p) \beta^{2} \mathcal{X}_{\omega}^{(1)}+(B \cdot p) \beta^{3} \mathcal{X}_{B}^{(1)}, \\
f^{(2)}=\mathcal{X}_{\Omega \Omega}+\mathcal{X}_{\Omega F}+\mathcal{X}_{F F},
\end{gathered}
$$

where

$$
\begin{aligned}
\mathcal{X}_{\Omega \Omega}= & \omega^{2} \beta^{2} \mathcal{X}_{\omega \omega 1}^{(2)}+\varepsilon^{2} \beta^{2} \mathcal{X}_{\varepsilon \varepsilon 1}^{(2)}+(\omega \cdot p)^{2} \beta^{4} \mathcal{X}_{\omega \omega 2}^{(2)} \\
& +(\varepsilon \cdot p)^{2} \beta^{4} \mathcal{X}_{\varepsilon \varepsilon 2}^{(2)}+\epsilon^{\nu \lambda \rho \sigma} u_{\nu} p_{\lambda} \omega_{\rho} \varepsilon_{\sigma} \beta^{3} \mathcal{X}_{\omega \varepsilon}^{(2)}, \\
\mathcal{X}_{\Omega F}= & \omega \cdot B \beta^{3} \mathcal{X}_{\omega B 1}^{(2)}+\varepsilon \cdot E \beta^{3} \mathcal{X}_{\varepsilon E 1}^{(2)}+(\omega \cdot p)(B \cdot p) \beta^{5} \mathcal{X}_{\omega B 2}^{(2)} \\
& +(\varepsilon \cdot p)(E \cdot p) \beta^{5} \mathcal{X}_{\varepsilon E 2}^{(2)}+\epsilon^{\nu \lambda \rho \sigma} u_{\nu} p_{\lambda} \omega_{\rho} E_{\sigma} \beta^{4} \mathcal{X}_{\omega E}^{(2)},
\end{aligned}
$$

$$
\begin{aligned}
\mathcal{X}_{F F}= & B^{2} \beta^{4} \mathcal{X}_{B B 1}^{(2)}+E^{2} \beta^{4} \mathcal{X}_{E E 1}^{(2)}+(B \cdot p)^{2} \beta^{6} \mathcal{X}_{B B 2}^{(2)} \\
& +(E \cdot p)^{2} \beta^{6} \mathcal{X}_{E E 2}^{(2)}+\epsilon^{\nu \lambda \rho \sigma} u_{\nu} p_{\lambda} B_{\rho} E_{\sigma} \beta^{5} \mathcal{X}_{B E}^{(2)},
\end{aligned}
$$

where all $\mathcal{X}$ functions depends on variables

$$
z=\beta \cdot p-\bar{\mu}_{s}, \quad \tilde{z}=\beta \cdot p+\bar{\mu}_{s} .
$$

From Eq. (3.2) at the first order, we have

$$
\begin{aligned}
0= & \nabla^{\mu}\left[p_{\mu} f^{(1)} \delta\left(p^{2}\right)\right], \\
= & \delta\left(p^{2}\right)\left[3(B \cdot p)(\varepsilon \cdot p)-(E \cdot p)(\omega \cdot p)+(\varepsilon \cdot B)(u \cdot p)^{2}\right. \\
& \left.+(\omega \cdot E) \bar{p}^{2}-2(u \cdot p) \epsilon_{\nu \lambda \rho \sigma} p^{\nu} u^{\lambda} \varepsilon^{\rho} E^{\sigma}\right] \beta^{3} \mathcal{X}_{B}^{(1)} \\
& +\delta\left(p^{2}\right)\left[(\omega \cdot E)(u \cdot p) \beta^{2} \mathcal{X}_{\omega}^{(1)}\right. \\
& \left.+\epsilon_{\mu \nu \rho \sigma} u^{\mu} p^{\nu} \omega^{\rho} B^{\sigma} \beta^{2} \mathcal{X}_{\omega}^{(1)}+(E \cdot B)(u \cdot p) \beta^{3} \mathcal{X}_{B}^{(1)}\right] \\
& -\delta\left(p^{2}\right)\left[(\omega \cdot p) \beta^{2} \partial_{\tilde{z}} \mathcal{X}_{\omega}^{(1)}+(B \cdot p) \beta^{4} \partial_{\tilde{z}} \mathcal{X}_{B}^{(1)}\right] 2 \beta(E \cdot p) .
\end{aligned}
$$

Obviously, this equation holds only if

$$
\mathcal{X}_{B}^{(1)}=\mathcal{X}_{\omega}^{(1)}=0,
$$

which means that there is no solution at the first order with nonvanishing $f^{(1)}$. However, it is interesting to note that when we turn off the electromagnetic field at the beginning, Eq. (7.7) holds automatically, so we have

$$
f^{(1)}=(\omega \cdot p) \beta^{2} \mathcal{X}_{\omega}^{(1)} .
$$

Hence the first order solution in Eq. (3.28) is a unique solution at global equilibrium. Similarly, Eq. (3.2) at the second order is 


$$
\begin{aligned}
0 & =\nabla^{\mu}\left[p_{\mu} f^{(2)} \delta\left(p^{2}\right)\right] \\
& =\delta\left(p^{2}\right) p_{\mu} \nabla^{\mu}\left[\mathcal{X}_{\Omega \Omega}+\mathcal{X}_{\Omega F}+\mathcal{X}_{F F}\right]
\end{aligned}
$$

We find that this equation holds only if following relations are fulfilled,

$$
\begin{gathered}
\mathcal{X}_{B B 1}^{(2)}=\mathcal{X}_{E E 1}^{(2)}=\mathcal{X}_{B B 2}^{(2)}=\mathcal{X}_{E E 2}^{(2)}=\mathcal{X}_{B E}^{(2)}=0, \\
\mathcal{X}_{\omega B 1}^{(2)}=\mathcal{X}_{\omega B 2}^{(2)}=\mathcal{X}_{\varepsilon E 2}^{(2)}=\mathcal{X}_{\omega E}^{(2)}=0 \\
X_{\omega \omega 2}^{(2)}=X_{\varepsilon \varepsilon 2}^{(2)}=0 \\
\mathcal{X}_{\varepsilon E 1}^{(2)}=\frac{1}{2} \mathcal{X}_{\omega \varepsilon}^{(2)}
\end{gathered}
$$

together with

$$
\begin{gathered}
\mathcal{X}_{\omega \varepsilon}^{(2)}=\frac{1}{\beta \cdot p}\left(\mathcal{X}_{\omega \omega 1}^{(2)}+\mathcal{X}_{\varepsilon \varepsilon 1}^{(2)}\right), \\
\mathcal{X}_{\omega \varepsilon}^{(2)}=2 \partial_{\tilde{z}} \mathcal{X}_{\varepsilon \varepsilon 1}^{(2)}, \\
\partial_{\tilde{z}} \mathcal{X}_{\omega \varepsilon}^{(2)}=\partial_{\tilde{z}} \mathcal{X}_{\omega \omega 1}^{(2)}=0 .
\end{gathered}
$$

It is easy to verify that the general solution must take the form of

$$
\begin{gathered}
\mathcal{X}_{\omega \omega 1}^{(2)}=a(z), \\
\mathcal{X}_{\omega \varepsilon}^{(2)}=b(z), \\
\mathcal{X}_{\varepsilon \varepsilon 1}^{(2)}=(\beta \cdot p) b(z)-a(z),
\end{gathered}
$$

where $a(z)$ and $b(z)$ are arbitrary real functions of $z$. Hence the general form of $f^{(2)}$ is

$$
\begin{aligned}
f^{(2)}= & \omega^{2} \beta^{2} a(z)+\varepsilon^{2} \beta^{2}[(\beta \cdot p) b(z)-a(z)] \\
& +\epsilon^{\nu \lambda \rho \sigma} u_{\nu} p_{\lambda} \omega_{\rho} \varepsilon_{\sigma} \beta^{3} b(z)+\frac{1}{2}(\varepsilon \cdot E) \beta^{3} b(z) .
\end{aligned}
$$

We see that there is no contribution from electromagnetic fields except a $\varepsilon \cdot E$ term. Integrating over momenta, we obtain contributions to the current and stress tensor

$$
\begin{aligned}
\Delta j_{\mu}^{(2)} & =\int d^{4} p f^{(2)} p_{\mu} \delta\left(p^{2}\right), \\
& =\left[A_{j} \omega^{2}+B_{j} \varepsilon^{2}+C_{j}(\varepsilon \cdot E)\right] u_{\mu}+D_{j} \epsilon_{\mu \nu \rho \sigma} u^{\nu} \omega^{\rho} \varepsilon^{\sigma},
\end{aligned}
$$

$$
\begin{aligned}
\Delta T_{\mu \nu}^{(2)}= & \int d^{4} p f^{(2)} p_{\mu} p_{\nu} \delta\left(p^{2}\right), \\
= & {\left[A_{T} \omega^{2}+B_{T} \varepsilon^{2}+C_{T}(\varepsilon \cdot E)\right]\left(u_{\mu} u_{\nu}-\frac{1}{3} \Delta_{\mu \nu}\right) } \\
& +D_{T}\left(u_{\mu} \epsilon_{\nu \lambda \rho \sigma}+u_{\nu} \epsilon_{\mu \lambda \rho \sigma}\right) u^{\lambda} \omega^{\rho} \varepsilon^{\sigma},
\end{aligned}
$$

where we have used $\Delta$ to denote these are additional contribution from nonvanishing $f^{(2)}$. After integration over $p_{0}$, these coefficients are

$$
\begin{gathered}
A_{j}=\frac{1}{2} \beta^{2} \int d^{3} p\left[a\left(z_{+}\right)-a\left(z_{-}\right)\right], \\
B_{j}=\frac{1}{2} \beta^{2} \int d^{3} p\left\{\beta p\left[b\left(z_{+}\right)+b\left(z_{-}\right)\right]-\left[a\left(z_{+}\right)-a\left(z_{-}\right)\right]\right\},
\end{gathered}
$$

$$
\begin{gathered}
C_{j}=\frac{1}{4} \beta^{3} \int d^{3} p\left[b\left(z_{+}\right)-b\left(z_{-}\right)\right], \\
D_{j}=\frac{1}{6} \beta^{3} \int d^{3} p p\left[b\left(z_{+}\right)+b\left(z_{-}\right)\right], \\
A_{T}=\frac{1}{2} \beta^{2} \int d^{3} p p\left[a\left(z_{+}\right)+a\left(z_{-}\right)\right],
\end{gathered}
$$

$B_{T}=\frac{1}{2} \beta^{2} \int d^{3} p p\left\{\beta p\left[b\left(z_{+}\right)-b\left(z_{-}\right)\right]-\left[a\left(z_{+}\right)+a\left(z_{-}\right)\right]\right\}$,

$$
\begin{aligned}
C_{T} & =\frac{1}{4} \beta^{3} \int d^{3} p p\left[b\left(z_{+}\right)+b\left(z_{-}\right)\right], \\
D_{T} & =\frac{1}{6} \beta^{3} \int d^{3} p p^{2}\left[b\left(z_{+}\right)-b\left(z_{-}\right)\right],
\end{aligned}
$$

where $z_{ \pm}=\beta \cdot p \mp \bar{\mu}_{s}$. Although unknown functions $a(z)$ and $b(z)$ are arbitrary, only two of these coefficients are independent. It is convenient to choose $A_{T}$ and $D_{T}$ as independent variables from which other coefficients can be derived through following relations

$$
\begin{gathered}
A_{j}=\frac{1}{3 \beta} \frac{\partial\left(\beta^{2} A_{T}\right)}{\partial \bar{\mu}_{s}}, \\
D_{j}=\frac{1}{4 \beta} \frac{\partial\left(\beta^{2} D_{T}\right)}{\partial \bar{\mu}_{s}}, \\
B_{j}=3 D_{j}-A_{j}, \\
C_{j}=\frac{1}{2} \frac{\partial\left(\beta D_{j}\right)}{\partial \bar{\mu}_{s}},
\end{gathered}
$$




$$
\begin{gathered}
C_{T}=\frac{3}{2} D_{j}, \\
B_{T}=3 D_{T}-A_{T} .
\end{gathered}
$$

In Refs. [68,69], the authors calculate the second order transport coefficients in global equilibrium without electromagnetic fields. We find that their results are different from our results in Eqs. (4.25), (4.26), and (5.26) from our special solution (3.31) with vanishing $f^{(1)}$ and $f^{(2)}$. The difference between our result and the result in Refs. [68,69] are given by

$$
\begin{aligned}
\Delta j_{s}^{\mu}= & \frac{\bar{\mu}_{s}}{8 \pi^{2} \beta}\left(\varepsilon^{2}+\omega^{2}\right) u^{\mu}+\frac{\bar{\mu}_{s}}{12 \pi^{2} \beta} \epsilon^{\mu \alpha \beta \gamma} u_{\alpha} \varepsilon_{\beta} \omega_{\gamma}, \\
\Delta T_{s}^{\mu \nu}= & \frac{\beta}{2} s \xi_{s}\left[\left(3 u^{\mu} u^{\nu}-\Delta^{\mu \nu}\right)\left(\frac{1}{2} \omega^{2}+\frac{5}{6} \varepsilon^{2}\right)\right. \\
& \left.+\frac{4}{3}\left(u^{\mu} \epsilon^{\nu \alpha \beta \gamma}+u^{\nu} \epsilon^{\mu \alpha \beta \gamma}\right) u_{\alpha} \omega_{\beta} \varepsilon_{\gamma}\right],
\end{aligned}
$$

with $\xi_{s}$ being given in Eq. (4.6). From these expressions, we can read off

$$
\begin{aligned}
& A_{T}=\frac{1}{16 \pi^{2} \beta^{2}}\left[\pi^{2}+3 \bar{\mu}_{s}^{2}\right], \\
& D_{T}=\frac{1}{18 \pi^{2} \beta^{2}}\left[\pi^{2}+3 \bar{\mu}_{s}^{2}\right] .
\end{aligned}
$$

Substituting it into the relations (7.32)-(7.37), we can obtain all other coefficients which are exactly consistent with the result of Refs. $[68,69]$ as well as $C_{j}=$ $1 / 24 \pi^{2}, C_{T}=\mu_{s} / 8 \pi^{2}$ for $\epsilon \cdot E$ terms when electromagnetic fields are involved. This implies that both results, ours and that of Refs. [68,69], are possible solutions which should correspond to two different density matrices.

\section{SUMMARY AND DISCUSSION}

We have derived in the covariant Wigner function formalism the charge and chiral currents as well as the stress tensor for chiral fermions in uniform vorticity and electromagnetic fields up to the second order of spatial derivatives. We present all possible second order contributions in quadratic forms of the vorticity and electromagnetic field. These contributions include coupling terms of electromagnetic-field-electromagnetic-field (ee), vorticityvorticity (vv), and vorticity-electromagnetic-field (ve). All the terms can modify the charge density, while only the "ee" and "vv" terms can modify the chiral charge density and "ve" term cannot contribute to it. We find that the electromagnetic field can induce Hall currents in the form $\epsilon^{\mu \nu \rho \sigma} u_{\nu} E_{\rho} B_{\sigma}$ in the charge and axial charge current. There is also a Hall term $\epsilon^{\mu \nu \rho \sigma} u_{\nu} E_{\rho} \omega_{\sigma}$ in the charge current. For the energy-momentum tensor at the second order, we find that the vorticity and electromagnetic field contribute to the energy density and pressure. The conservation laws as well as chiral and trace anomaly can be verified with our second order solution. All coefficients we obtain in this work can be directly applied to the anomalous hydrodynamics as inputs. We also demonstrate that the solution in global equilibrium is fully constrained at the first order while solutions at the second order can only be constrained up to some unknown functions. These unknown functions appear in the "vv" part and $\varepsilon \cdot E$ term in the "ve" part. Other contributions can be fully constrained.

In this work, we restrict ourselves to a noninteracting chiral system without collision terms under the constant electromagnetic and vorticity field. If we go beyond to include collision terms but still in global equilibrium, collision terms will not change present results since collision terms always vanish in global equilibrium. Instead, if we consider nonequilibrium and varying fields, our present results still provide a baseline for studying these effects. We can expand the solution around the results in global equilibrium given here and investigate contributions from collisions or variation of fields. We reserve the topics along this line in a future study.

\section{ACKNOWLEDGMENTS}

J. H. G. would like to thank Shu Lin for helpful discussion. This work was supported in part by the National Natural Science Foundation of China under Grants No. 11890713, No. 11675092, No. 11535012, and No. 11947301, and the Natural Science Foundation of Shandong Province under Grant No. JQ201601.

\section{APPENDIX A: INTEGRATION IN $T_{s, \mathrm{ee}}^{(2) \mu \nu}$}

In this Appendix, we give detailed derivations of some integrals in $T_{s, \mathrm{ee}}^{(2) \mu \nu}$. All other coefficients in $j_{s}^{(2)}, T_{s, \mathrm{Vv}}^{(2) \mu \nu}$, and $T_{s, v e}^{(2) \mu \nu}$ can be derived in a similar way. We take the integral in the first term of Eq. (5.20) as an example,

$$
I \equiv \int d^{4-\epsilon} p(u \cdot p)^{4} f \delta^{\prime \prime \prime}\left(p^{2}\right) .
$$

First, we need to integrate out the $\delta$ function by using the identity

$$
\begin{aligned}
\delta^{\prime \prime \prime}\left(p^{2}\right)= & \frac{1}{16 p_{0}|\vec{p}|^{3}}\left[\delta^{\prime \prime \prime}\left(p_{0}-|\vec{p}|\right)+\delta^{\prime \prime \prime}\left(p_{0}+|\vec{p}|\right)\right] \\
& +\frac{3}{16 p_{0}|\vec{p}|^{4}}\left[\delta^{\prime \prime}\left(p_{0}-|\vec{p}|\right)-\delta^{\prime \prime}\left(p_{0}+|\vec{p}|\right)\right] \\
& +\frac{3}{16 p_{0}|\vec{p}|^{5}}\left[\delta^{\prime}\left(p_{0}-|\vec{p}|\right)+\delta^{\prime}\left(p_{0}+|\vec{p}|\right)\right],
\end{aligned}
$$

and obtain 


$$
\begin{aligned}
I & =-\frac{\pi^{(3-\epsilon) / 2}}{8 \Gamma((3-\epsilon) / 2)} \int \frac{d|\vec{p}|}{|\vec{p}|^{\epsilon}}\left[\frac{1}{|\vec{p}|} \frac{d^{3}}{d|\vec{p}|^{3}}-\frac{3}{|\vec{p}|^{2}} \frac{d^{2}}{d|\vec{p}|^{2}}+\frac{3}{|\vec{p}|^{3}} \frac{d}{d|\vec{p}|}\right]\left[|\vec{p}|^{3}\left(f^{+}+f^{-}\right)\right] \\
& =-\frac{\pi^{(3-\epsilon) / 2}}{8 \Gamma((3-\epsilon) / 2)} \int \frac{d|\vec{p}|}{|\vec{p}|^{\epsilon}}\left[|\vec{p}|^{2} \frac{d^{3}}{d|\vec{p}|^{3}}+6|\vec{p}| \frac{d^{2}}{d|\vec{p}|^{2}}+3 \frac{d}{d|\vec{p}|}-3 \frac{1}{|\vec{p}|}\right]\left(f^{+}+f^{-}\right),
\end{aligned}
$$

where the prefactor is from the integration over the solid angle in $4-\epsilon$ dimension and $f^{+} / f^{-}$denotes the contribution from $p_{0}>0 / p_{0}<0$ in Eqs. (3.11)/(3.12). After integration by parts, we have

$$
\begin{aligned}
I & =\frac{3 \pi^{(3-\epsilon) / 2}}{8 \Gamma((3-\epsilon) / 2)} \int d|\vec{p}|\left[\frac{1-3 \epsilon}{3|\vec{p}|^{\epsilon}} \frac{d}{d|\vec{p}|}+\frac{1}{|\vec{p}|^{1+\epsilon}}\right]\left(f^{+}+f^{-}\right), \\
& =\frac{3 \pi^{(3-\epsilon) / 2}}{8 \Gamma((3-\epsilon) / 2)}\left(\frac{\epsilon}{3}+1\right) \int d|\vec{p}| \frac{1}{|\vec{p}|^{1+\epsilon}}\left(f^{+}+f^{-}\right), \\
& \equiv \frac{(3+\epsilon)}{16} \kappa_{s}^{\epsilon},
\end{aligned}
$$

where

$$
\begin{aligned}
\kappa_{s}^{\epsilon} & =\frac{2 \pi^{(3-\epsilon) / 2}}{\Gamma((3-\epsilon) / 2)} \int \frac{d|\vec{p}|}{|\vec{p}|^{1+\epsilon}}\left(f^{+}+f^{-}\right), \\
& =\frac{4 \pi^{(3-\epsilon) / 2} T^{-\epsilon}}{\Gamma((3-\epsilon) / 2)(2 \pi)^{3-\epsilon}} \int_{0}^{\infty} \frac{d y}{y^{1+\epsilon}}\left[\frac{1}{e^{y-\mu_{s}}+1}+\frac{1}{e^{y+\bar{\mu}_{s}}+1}-1\right] .
\end{aligned}
$$

It should be noted that if we did not include the vacuum contribution -1 in the square brackets of the last line, the integral would have infrared divergence. Once we include it as above, the infrared divergence is canceled with only the ultraviolet divergence left. We can single out the divergence by another integration by parts

$$
\kappa_{s}^{\epsilon}=-\frac{4 \pi^{\frac{3-\epsilon}{2}} T^{-\epsilon}}{\Gamma\left(\frac{3-\epsilon}{2}\right)(2 \pi)^{3-\epsilon}}\left\{\frac{1}{\epsilon}-\int_{0}^{\infty} d y \ln y\left[\frac{e^{y-\bar{\mu}_{s}}}{\left(e^{y-\bar{\mu}_{s}}+1\right)^{2}}+\frac{e^{y+\bar{\mu}_{s}}}{\left(e^{y+\bar{\mu}_{s}}+1\right)^{2}}\right]\right\} .
$$

The $1 / \epsilon$ pole term corresponds to a logarithmic divergence in the momentum integral.

\section{APPENDIX B: RESULTS IN THE LANDAU FRAME}

In relativistic hydrodynamics, one has a freedom to choose any frame characterized by a different fluid velocity. The Landau frame is the one in which the fluid velocity satisfies $u_{\mu} T^{\mu \nu}=\rho u^{\nu}$. Since the energy momentum tensor in Sec. V has an antisymmetric part in the first and second order, in this section, we will rewrite the symmetric part of the stress tensor up to the second order in the Landau frame. Let us introduce the fluid velocity $U^{\mu}$ in the Landau frame given by

$$
\begin{aligned}
U^{\mu}= & u^{\mu}+\frac{n_{5}}{\rho+P} \omega^{\mu}+\frac{\xi}{2(\rho+P)} B^{\mu}-\left[\frac{n_{5}^{2}}{2(\rho+P)^{2}} \omega^{2}+\frac{\xi^{2}}{8(\rho+P)^{2}} B^{2}+\frac{n_{5} \xi}{2(\rho+P)^{2}} \omega \cdot B\right] u^{\mu} \\
& +\frac{\xi_{5}}{\rho+P} \epsilon^{\mu \alpha \beta \gamma} u_{\alpha} \varepsilon_{\beta} \omega_{\gamma}+\frac{\xi_{B 5}}{2(\rho+P)} \epsilon^{\mu \alpha \beta \gamma} u_{\alpha} E_{\beta} \omega_{\gamma}+\frac{\kappa_{4}}{\rho+P} \epsilon^{\mu \alpha \beta \gamma} u_{\alpha} E_{\beta} B_{\gamma} .
\end{aligned}
$$

It is easy to verify that $U^{2}=1$ up to the second order. From this relation, we can also express $u^{\mu}$ in terms of $U^{\mu}$,

$$
\begin{aligned}
u^{\mu}= & U^{\mu}-\frac{n_{5}}{\rho+P} \omega_{U}^{\mu}-\frac{\xi}{2(\rho+P)} B_{U}^{\mu}-\left[\frac{n_{5}^{2}}{2(\rho+P)^{2}} \omega_{U}^{2}+\frac{\xi^{2}}{8(\rho+P)^{2}} B_{U}^{2}+\frac{n_{5} \xi}{2(\rho+P)^{2}} \omega_{U} \cdot B_{U}\right] U^{\mu} \\
& -\left[\frac{\xi_{5}}{\rho+P}+\frac{n_{5}^{2}}{(\rho+P)^{2}}\right] \epsilon^{\mu \alpha \beta \gamma} U_{\alpha} \varepsilon_{U \beta} \omega_{U \gamma}-\left[\frac{\xi_{B 5}}{2(\rho+P)}+\frac{n_{5} \xi}{(\rho+P)^{2}}\right] \epsilon^{\mu \alpha \beta \gamma} U_{\alpha} E_{U \beta} \omega_{U \gamma} \\
& -\left[\frac{\kappa_{4}}{\rho+P}+\frac{\xi^{2}}{4(\rho+P)^{2}}\right] \epsilon^{\mu \alpha \beta \gamma} U_{\alpha} E_{U \beta} B_{U \gamma},
\end{aligned}
$$


where $\omega_{U}^{\mu}=T \tilde{\Omega}^{\mu \nu} U_{\nu}, \varepsilon_{U}^{\mu}=T \Omega^{\mu \nu} U_{\nu}, B_{U}^{\mu}=\tilde{F}^{\mu \nu} U_{\nu}$, and $E_{U}^{\mu}=F^{\mu \nu} U_{\nu}$ are counterparts of $\omega^{\mu}, \varepsilon^{\mu}, B^{\mu}$, and $E^{\mu}$ in the Landau frame, respectively. Up to the second order, the corresponding relations between two groups of quantities are given by

$$
\begin{gathered}
\omega^{\mu}=\omega_{U}^{\mu}+\left(\frac{n_{5}}{\rho+P} \omega_{U}^{2}+\frac{\xi}{2(\rho+P)} \omega_{U} \cdot B_{U}\right) U^{\mu} \\
+\frac{n_{5}}{\rho+P} \epsilon^{\mu \nu \alpha \beta} \omega_{U \nu} U_{\alpha} \varepsilon_{U \beta}+\frac{\xi}{2(\rho+P)} \epsilon^{\mu \nu \alpha \beta} B_{U \nu} U_{\alpha} \varepsilon_{U \beta}, \\
\varepsilon^{\mu}=\varepsilon_{U}^{\mu}+\left(\frac{n_{5}}{\rho+P} \varepsilon_{U} \cdot \omega_{U}+\frac{\xi}{2(\rho+P)} \varepsilon_{U} \cdot B_{U}\right) U^{\mu}-\frac{\xi}{2(\rho+P)} \epsilon^{\mu \nu \alpha \beta} B_{U \nu} U_{\alpha} \omega_{U \beta}, \\
B^{\mu}=B_{U}^{\mu}+\left(\frac{n_{5}}{\rho+P} \omega_{U} \cdot B_{U}+\frac{\xi}{2(\rho+P)} B_{U}^{2}\right) U^{\mu} \\
+\frac{n_{5}}{\rho+P} \epsilon^{\mu \nu \alpha \beta} \omega_{U \nu} U_{\alpha} E_{U \beta}+\frac{\xi}{2(\rho+P)} \epsilon^{\mu \nu \alpha \beta} B_{U \nu} U_{\alpha} E_{U \beta}, \\
E^{\mu}=E_{U}^{\mu}+\left(\frac{n_{5}}{\rho+P} E_{U} \cdot \omega_{U}+\frac{\xi}{2(\rho+P)} E_{U} \cdot B_{U}\right) U^{\mu}-\frac{n_{5}}{\rho+P} \epsilon^{\mu \nu \alpha \beta} \omega_{U \nu} U_{\alpha} B_{U \beta} .
\end{gathered}
$$

Hence the difference between frames arises only at the second order. With these equations, we can obtain the symmetric stress tensor in the Landau frame

$$
\begin{aligned}
T^{\mu \nu}= & \left\{\rho+\left(\frac{n_{5}^{2}}{\rho+P}-\frac{3}{2} \xi_{5}\right) \omega_{U}^{2}+\left[\frac{\xi^{2}}{4(\rho+P)}+\kappa_{1}^{B}\right] B_{U}^{2}+\left(\frac{n_{5} \xi}{\rho+P}-\frac{\xi_{B 5}}{2}\right) \omega_{U} \cdot B_{U}-\frac{3}{2} \xi_{5} \varepsilon_{U}^{2}-\frac{1}{2} \xi_{B 5} \varepsilon_{U} \cdot E_{U}+\kappa_{1}^{E} E_{U}^{2}\right\} U^{\mu} U^{\nu} \\
& -\left[P-\frac{1}{2} \xi_{5}\left(\omega_{U}^{2}+\varepsilon_{U}^{2}\right)-\kappa_{2}^{E} E_{U}^{2}-\kappa_{2}^{B} B_{U}^{2}\right] \Delta_{U}^{\mu \nu}+\left(E_{U}^{\mu} E_{U}^{\nu}+B_{U}^{\mu} B_{U}^{\nu}\right) \kappa_{3}+\frac{1}{2} \xi_{B 5}\left(\omega_{U}^{\mu} B_{U}^{\nu}+E_{U}^{\mu} \varepsilon_{U}^{\nu}\right) \\
& -\frac{n_{5}^{2}}{\rho+P} \omega_{U}^{\mu} \omega_{U}^{\nu}-\frac{\xi^{2}}{4(\rho+P)} B_{U}^{\mu} B_{U}^{\nu}-\frac{n_{5} \xi}{2(\rho+P)}\left(\omega_{U}^{\mu} B_{U}^{\nu}+\omega_{U}^{\nu} B_{U}^{\mu}\right),
\end{aligned}
$$

where $\Delta_{U}^{\mu \nu}=g^{\mu \nu}-U^{\mu} U^{\nu}$. The vector current in the Landau frame is given by

$$
\begin{aligned}
j^{\mu}= & n U^{\mu}+\left(\xi-\frac{n n_{5}}{\rho+P}\right) \omega_{U}^{\mu}+\left(\xi_{B}-\frac{n \xi}{2(\rho+P)}\right) B_{U}^{\mu}-\left[\frac{n n_{5}^{2}}{2(\rho+P)^{2}}-\frac{\xi n_{5}}{\rho+P}\right] \omega_{U}^{2} U^{\mu}+\left[\frac{\xi_{B} \xi}{2(\rho+P)}-\frac{n \xi^{2}}{8(\rho+P)^{2}}\right] B_{U}^{2} U^{\mu} \\
& -\left[\frac{n n_{5} \xi}{2(\rho+P)^{2}}-\frac{\xi^{2}}{2(\rho+P)}-\frac{\xi_{B} n_{5}}{\rho+P}\right] \omega_{U} \cdot B_{U} U^{\mu}-\left(\frac{n \xi_{5}}{\rho+P}+\frac{n n_{5}^{2}}{(\rho+P)^{2}}-\frac{\xi n_{5}}{\rho+P}\right) \epsilon^{\mu \alpha \beta \gamma} U_{\alpha} \varepsilon_{U \beta} \omega_{U \gamma} \\
& +\left[\frac{\xi_{B} \xi}{2(\rho+P)}-\frac{n \xi^{2}}{4(\rho+P)^{2}}-\frac{n \kappa_{4}}{\rho+P}\right] \epsilon^{\mu \alpha \beta \gamma} U_{\alpha} E_{U \beta} B_{U \gamma}+\left[\frac{\xi^{2}}{2(\rho+P)}-\frac{n n_{5} \xi}{2(\rho+P)^{2}}\right] \epsilon^{\mu \alpha \beta \gamma} U_{\alpha} \varepsilon_{U \beta} B_{U \gamma} \\
& -\left[\frac{n \xi_{B 5}}{2(\rho+P)}+\frac{n n_{5} \xi}{2(\rho+P)^{2}}-\frac{\xi_{B} n_{5}}{\rho+P}\right] \epsilon^{\mu \alpha \beta \gamma} U_{\alpha} E_{U \beta} \omega_{U \gamma} \\
& -\xi_{B 5}\left(\varepsilon_{U}^{2}+\omega_{U}^{2}\right) U^{\mu}-\frac{1}{4 \pi^{2}}\left[\left(\omega_{U} \cdot B_{U}+\varepsilon_{U} \cdot E_{U}\right) U^{\mu}+\varepsilon^{\mu \nu \rho \sigma} U_{\nu} E_{U \rho} \omega_{U \sigma}\right] \\
& -\frac{C}{12 \pi^{2}}\left[U^{\mu}\left(E_{U}^{2}+B_{U}^{2}\right)+2 \varepsilon^{\mu \nu \rho \sigma} U_{\nu} E_{U \rho} B_{U \sigma}\right] .
\end{aligned}
$$

The axial current in the Landau frame is given by 


$$
\begin{aligned}
j_{5}^{\mu}= & n_{5} U^{\mu}+\left(\xi_{5}-\frac{n_{5}^{2}}{\rho+P}\right) \omega_{U}^{\mu}+\left(\xi_{B 5}-\frac{n_{5} \xi}{2(\rho+P)}\right) B_{U}^{\mu} \\
& -\left[\frac{n_{5}^{3}}{2(\rho+P)^{2}}-\frac{\xi_{5} n_{5}}{\rho+P}\right] \omega_{U}^{2} U^{\mu}+\left[\frac{\xi_{B 5} \xi}{2(\rho+P)}-\frac{n_{5} \xi^{2}}{8(\rho+P)^{2}}\right] B_{U}^{2} U^{\mu} \\
& -\left[\frac{n_{5}^{2} \xi}{2(\rho+P)^{2}}-\frac{\xi_{5} \xi}{2(\rho+P)}-\frac{\xi_{B 5} n_{5}}{\rho+P}\right] \omega_{U} \cdot B_{U} U^{\mu} \\
& -\frac{n_{5}^{3}}{(\rho+P)^{2}} \epsilon^{\mu \alpha \beta \gamma} U_{\alpha} \varepsilon_{U \beta} \omega_{U \gamma}+\left[\frac{\xi_{B 5} \xi}{2(\rho+P)}-\frac{n_{5} \kappa_{4}}{\rho+P}-\frac{n_{5} \xi^{2}}{4(\rho+P)^{2}}\right] \epsilon^{\mu \alpha \beta \gamma} U_{\alpha} E_{U \beta} B_{U \gamma} \\
& +\left[\frac{\xi_{5} \xi}{2(\rho+P)}-\frac{n_{5}^{2} \xi}{2(\rho+P)^{2}}\right] \epsilon^{\mu \alpha \beta \gamma} U_{\alpha} \varepsilon_{U \beta} B_{U \gamma}+\left[\frac{n_{5} \xi_{B 5}}{2(\rho+P)}-\frac{n_{5}^{2} \xi}{2(\rho+P)^{2}}\right] \epsilon^{\mu \alpha \beta \gamma} U_{\alpha} E_{U \beta} \omega_{U \gamma} \\
& -\xi_{B}\left(\varepsilon_{U}^{2}+\omega_{U}^{2}\right) U^{\mu}-\frac{C_{5}}{12 \pi^{2}}\left[\left(E_{U}^{2}+B_{U}^{2}\right) U^{\mu}+2 \epsilon^{\mu \nu \rho \sigma} U_{\nu} E_{U \rho U} B_{U \sigma}\right] .
\end{aligned}
$$

[1] A. Vilenkin, Phys. Rev. D 22, 3080 (1980).

[2] D. E. Kharzeev, L. D. McLerran, and H. J. Warringa, Nucl. Phys. A803, 227 (2008).

[3] K. Fukushima, D. E. Kharzeev, and H. J. Warringa, Phys. Rev. D 78, 074033 (2008).

[4] A. Vilenkin, Phys. Lett. 80B, 150 (1978).

[5] D. Kharzeev and A. Zhitnitsky, Nucl. Phys. A797, 67 (2007).

[6] J. Erdmenger, M. Haack, M. Kaminski, and A. Yarom, J. High Energy Phys. 01 (2009) 055.

[7] N. Banerjee, J. Bhattacharya, S. Bhattacharyya, S. Dutta, R. Loganayagam, and P. Surowka, J. High Energy Phys. 01 (2011) 094.

[8] D. T. Son and A. R. Zhitnitsky, Phys. Rev. D 70, 074018 (2004).

[9] M. A. Metlitski and A. R. Zhitnitsky, Phys. Rev. D 72, 045011 (2005).

[10] J. H. Gao, Z. T. Liang, S. Pu, Q. Wang, and X. N. Wang, Phys. Rev. Lett. 109, 232301 (2012).

[11] G. M. Newman, J. High Energy Phys. 01 (2006) 158.

[12] H. U. Yee, J. High Energy Phys. 11 (2009) 085.

[13] A. Rebhan, A. Schmitt, and S. A. Stricker, J. High Energy Phys. 01 (2010) 026.

[14] A. Gorsky, P. N. Kopnin, and A. V. Zayakin, Phys. Rev. D 83, 014023 (2011).

[15] A. Gynther, K. Landsteiner, F. Pena-Benitez, and A. Rebhan, J. High Energy Phys. 02 (2011) 110.

[16] C. Hoyos, T. Nishioka, and A. O'Bannon, J. High Energy Phys. 10 (2011) 084.

[17] I. Amado, K. Landsteiner, and F. Pena-Benitez, J. High Energy Phys. 05 (2011) 081.

[18] V. P. Nair, R. Ray, and S. Roy, Phys. Rev. D 86, 025012 (2012).

[19] T. Kalaydzhyan and I. Kirsch, Phys. Rev. Lett. 106, 211601 (2011).

[20] S. Lin and H. U. Yee, Phys. Rev. D 88, 025030 (2013).
[21] D. T. Son and P. Surowka, Phys. Rev. Lett. 103, 191601 (2009).

[22] A. V. Sadofyev and M. V. Isachenkov, Phys. Lett. B 697, 404 (2011).

[23] S. Pu, J. H. Gao, and Q. Wang, Phys. Rev. D 83, 094017 (2011).

[24] D. E. Kharzeev and H.-U. Yee, Phys. Rev. D 84, 045025 (2011).

[25] D. E. Kharzeev and H. J. Warringa, Phys. Rev. D 80, 034028 (2009).

[26] K. Fukushima, D. E. Kharzeev, and H. J. Warringa, Nucl. Phys. A836, 311 (2010).

[27] M. Asakawa, A. Majumder, and B. Muller, Phys. Rev. C 81, 064912 (2010).

[28] K. Fukushima, D. E. Kharzeev, and H. J. Warringa, Phys. Rev. Lett. 104, 212001 (2010).

[29] K. Fukushima and M. Ruggieri, Phys. Rev. D 82, 054001 (2010).

[30] K. Landsteiner, E. Megias, and F. Pena-Benitez, Phys. Rev. Lett. 107, 021601 (2011).

[31] D. Hou, H. Liu, and H.c. Ren, J. High Energy Phys. 05 (2011) 046.

[32] D. F. Hou, H. Liu, and H. c. Ren, Phys. Rev. D 86, 121703 (2012).

[33] S. Lin and L. Yang, Phys. Rev. D 98, 114022 (2018).

[34] B. Feng, D. F. Hou, and H. C. Ren, Phys. Rev. D 99, 036010 (2019).

[35] R. D. Dong, R. H. Fang, D. F. Hou, and D. She, Chin. Phys. C 44, 074106 (2020).

[36] M. A. Stephanov and Y. Yin, Phys. Rev. Lett. 109, 162001 (2012).

[37] D. T. Son and N. Yamamoto, Phys. Rev. D 87, 085016 (2013).

[38] J. W. Chen, S. Pu, Q. Wang, and X. N. Wang, Phys. Rev. Lett. 110, 262301 (2013). 
[39] C. Manuel and J. M. Torres-Rincon, Phys. Rev. D 89, 096002 (2014).

[40] J. Y. Chen, D. T. Son, M. A. Stephanov, H. U. Yee, and Y. Yin, Phys. Rev. Lett. 113, 182302 (2014).

[41] J. Y. Chen, D. T. Son, and M. A. Stephanov, Phys. Rev. Lett. 115, 021601 (2015).

[42] Y. Hidaka, S. Pu, and D. L. Yang, Phys. Rev. D 95, 091901 (2017).

[43] N. Mueller and R. Venugopalan, Phys. Rev. D 97, 051901 (2018).

[44] A. Huang, Y. Jiang, S. Shi, J. Liao, and P. Zhuang, Phys. Lett. B 777, 177 (2018).

[45] A. Huang, S. Shi, Y. Jiang, J. Liao, and P. Zhuang, Phys. Rev. D 98, 036010 (2018).

[46] Y. Hidaka and D. L. Yang, Phys. Rev. D 98, 016012 (2018).

[47] J. H. Gao, Z. T. Liang, Q. Wang, and X. N. Wang, Phys. Rev. D 98, 036019 (2018).

[48] J. H. Gao, J. Y. Pang, and Q. Wang, Phys. Rev. D 100, 016008 (2019).

[49] Y. C. Liu, L. L. Gao, K. Mameda, and X. G. Huang, Phys. Rev. D 99, 085014 (2019).

[50] I. Muller, Z. Phys. 198, 329 (1967).

[51] W. Israel, Ann. Phys. (N.Y.) 100, 310 (1976).

[52] W. Israel and J. Stewart, Ann. Phys. (N.Y.) 118, 341 (1979).

[53] W. Hiscock and L. Lindblom, Ann. Phys. (N.Y.) 151, 466 (1983).

[54] W. A. Hiscock and L. Lindblom, Phys. Rev. D 31, 725 (1985).

[55] W. A. Hiscock and L. Lindblom, Phys. Rev. D 35, 3723 (1987).

[56] A. Bzdak and V. Skokov, Phys. Lett. B 710, 171 (2012).

[57] W. T. Deng and X. G. Huang, Phys. Rev. C 85, 044907 (2012).

[58] J. Bloczynski, X. G. Huang, X. Zhang, and J. Liao, Phys. Lett. B 718, 1529 (2013).

[59] Z. T. Liang and X. N. Wang, Phys. Rev. Lett. 94, 102301 (2005); 96, 039901(E) (2006).

[60] J. H. Gao, S. W. Chen, W. t. Deng, Z. T. Liang, Q. Wang, and X. N. Wang, Phys. Rev. C 77, 044902 (2008).

[61] F. Becattini, F. Piccinini, and J. Rizzo, Phys. Rev. C 77, 024906 (2008).

[62] L. P. Csernai, V. K. Magas, and D. J. Wang, Phys. Rev. C 87, 034906 (2013).

[63] Y. Jiang, Z. W. Lin, and J. Liao, Phys. Rev. C 94, 044910 (2016); 95, 049904(E) (2017).
[64] W. T. Deng and X. G. Huang, Phys. Rev. C 93, 064907 (2016).

[65] L. G. Pang, H. Petersen, Q. Wang, and X. N. Wang, Phys. Rev. Lett. 117, 192301 (2016).

[66] A. Jimenez-Alba and H. U. Yee, Phys. Rev. D 92, 014023 (2015).

[67] K. Hattori and Y. Yin, Phys. Rev. Lett. 117, 152002 (2016).

[68] M. Buzzegoli, E. Grossi, and F. Becattini, J. High Energy Phys. 10 (2017) 091; 07 (2018) 119(E).

[69] M. Buzzegoli and F. Becattini, J. High Energy Phys. 12 (2018) 002.

[70] D. Satow, Phys. Rev. D 90, 034018 (2014).

[71] E. V. Gorbar, V. A. Miransky, I. A. Shovkovy, and P. O. Sukhachov, Phys. Rev. B 95, 205141 (2017).

[72] E. V. Gorbar, D. O. Rybalka, and I. A. Shovkovy, Phys. Rev. D 95, 096010 (2017).

[73] N. Abbasi, F. Taghinavaz, and O. Tavakol, J. High Energy Phys. 03 (2019) 051.

[74] N. Banerjee, J. Bhattacharya, S. Bhattacharyya, S. Jain, S. Minwalla, and T. Sharma, J. High Energy Phys. 09 (2012) 046.

[75] S. Bhattacharyya, J. R. David, and S. Thakur, J. High Energy Phys. 01 (2014) 010.

[76] E. Megias and M. Valle, J. High Energy Phys. 11 (2014) 005.

[77] Y. Bu and S. Lin, Eur. Phys. J. C 80, 401 (2020).

[78] U. W. Heinz, Phys. Rev. Lett. 51, 351 (1983).

[79] H. T. Elze, M. Gyulassy, and D. Vasak, Nucl. Phys. B276, 706 (1986).

[80] D. Vasak, M. Gyulassy, and H. T. Elze, Ann. Phys. (N.Y.) 173, 462 (1987).

[81] P. Zhuang and U. W. Heinz, Ann. Phys. (N.Y.) 245, 311 (1996).

[82] J. h. Gao and Q. Wang, Phys. Lett. B 749, 542 (2015).

[83] J.h. Gao, S. Pu, and Q. Wang, Phys. Rev. D 96, 016002 (2017).

[84] J. H. Gao, Z. T. Liang, and Q. Wang, Phys. Rev. D 101, 096015 (2020).

[85] X. L. Sheng, D. H. Rischke, D. Vasak, and Q. Wang, Eur. Phys. J. A 54, 21 (2018).

[86] X. L. Sheng, R. H. Fang, Q. Wang, and D. H. Rischke, Phys. Rev. D 99, 056004 (2019).

[87] M. E. Peskin and D. V. Schroeder, An Introduction to Quantum Field Theory (Addison-Wesley, Reading, USA, 1995). 